TRANSACTIONS OF THE

AMERICAN MATHEMATICAL SOCIETY

Volume 362, Number 4, April 2010, Pages 2167-2188

S 0002-9947(09)04941-1

Article electronically published on October 19, 2009

\title{
FUNDAMENTAL GROUPS OF GALOIS CLOSURES OF GENERIC PROJECTIONS
}

\author{
CHRISTIAN LIEDTKE
}

\begin{abstract}
For the Galois closure $X_{\text {gal }}$ of a generic projection from a surface $X$, it is believed that $\pi_{1}\left(X_{\text {gal }}\right)$ gives rise to new invariants of $X$. However, in all examples this group is surprisingly simple. In this article, we offer an explanation for this phenomenon: We compute a quotient of $\pi_{1}\left(X_{\text {gal }}\right)$ that depends on $\pi_{1}(X)$ and data from the generic projection only. In all known examples, this quotient is in fact isomorphic to $\pi_{1}\left(X_{\text {gal }}\right)$. As a byproduct, we simplify the computations of Moishezon, Teicher and others.
\end{abstract}

\section{INTRODUCTION}

One approach to a fine classification of algebraic surfaces studies generic projections. For that one embeds a given surface $X$ into some large projective space and projects it generically onto a plane. If $D$ is the branch curve of such a projection, it is possible to recover $X$ from the fundamental group $\pi_{1}\left(\mathbb{P}^{2}-D\right)$ and a little bit of extra data. Even though these fundamental groups are extremely difficult to compute, Moishezon $\mathrm{Mo}$ and later Teicher and others developed tools ("braid monodromy factorisations") to attack these computations systematically. Eventually, one hopes to obtain new invariants of surfaces from these groups.

One of these invariants of surfaces, which has itself a geometric interpretation, is the fundamental group of the corresponding Galois closure: The Galois closure $X_{\text {gal }}$ attached to a generic projection from $X$ is again a smooth projective surface. Its fundamental group $\pi_{1}\left(X_{\text {gal }}\right)$ is a subquotient of $\pi_{1}\left(\mathbb{P}^{2}-D\right)$, and therefore it is expected to contain non-trivial information about the surface $X$.

Typically, $X_{\text {gal }}$ is a surface of general type. Miyaoka Mi proved that there are many surfaces with positive index among Galois closures of generic projections, i.e., their Chern numbers fulfil $c_{1}{ }^{2}>2 c_{2}$. The "watershed-conjecture", attributed to Bogomolov, stated that surfaces of general type with positive index have infinite fundamental groups. However, when Moishezon and Teicher MoTe1 first computed $\pi_{1}\left(X_{\text {gal }}\right)$ for generic projections from $\mathbb{P}^{1} \times \mathbb{P}^{1}$ they found simply connected surfaces of general type with positive index among them. These were the first counterexamples to this conjecture. Their computations are quite involved and use degeneration techniques, braid monodromy factorisations and much combinatorial group theory.

Since then, the groups $\pi_{1}\left(X_{\text {gal }}\right)$ have been computed for many more examples. For generic projections from minimal rational surfaces (except the Veronese surface $\left.V_{4}\right)$ the group $\pi_{1}\left(X_{\text {gal }}\right)$ is always finite Abelian. Also, certain generic projections

Received by the editors November 2, 2005, and, in revised form, June 9, 2008.

2000 Mathematics Subject Classification. Primary 14E20, 14J29.

(C)2009 American Mathematical Society 2167

Reverts to public domain 28 years from publication 
from $\mathbb{P}^{1} \times E$, where $E$ is an elliptic curve, have been worked out. Here, the groups $\pi_{1}\left(X_{\text {gal }}\right)$ are close to being free Abelian. Why do these groups have such a simple structure?

Here, we give an explanation: Let $X_{\text {gal }}$ be the Galois closure of a generic projection $f: X \rightarrow \mathbb{P}^{2}$. We let $\mathcal{L}:=f^{*}\left(\mathcal{O}_{\mathbb{P}^{2}}(1)\right)$ be the very ample line bundle that defines $f$. We will assume that the degree $n:=\operatorname{deg} f$ is at least 5 .

In Theorem 3.6 and Theorem 4.5] we determine the structure of $\pi_{1}\left(X_{\mathrm{gal}}\right) / C^{\mathrm{proj}}$,

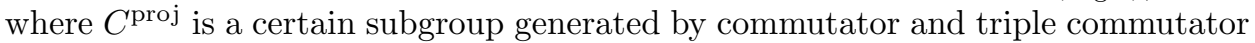
relations. Since our structure results need more notation and are a little bit messy to state, we first give a few corollaries.

Corollary 4.6. If $\pi_{1}(X)$ is finite, then so is $\pi_{1}\left(X_{\text {gal }}\right) / C^{\text {proj }}$.

Corollary 4.9, The rank of $H_{1}\left(X_{\text {gal }}, \mathbb{Z}\right) / \overline{C^{\text {proj }}}$ as an Abelian group is equal to $(n-1)$ times the rank of $H_{1}(X, \mathbb{Z})$.

Proposition 4.11. Let $X$ be a simply-connected surface. Let d be the divisibility index of $\mathcal{L}$ in $\operatorname{Pic}(X)$. Then there exists an isomorphism

$$
\pi_{1}\left(X_{\text {gal }}\right) / C^{\text {proj }} \cong(\mathbb{Z} / d \mathbb{Z})^{n-2} .
$$

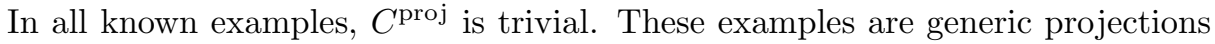
from a minimal rational surface, which have been computed by Moishezon, Teicher and Robb ([MoTe1], MoTe2], MTR]), and certain generic projections from $E \times \mathbb{P}^{1}$, where $E$ is an elliptic curve, which have been computed by Amram, Goldberg, Teicher and Vishne ([AG], AGTV]). We discuss these examples in Section [5 In particular, we compute $\pi_{1}\left(X_{\text {gal }}\right)$ modulo $C^{\text {proj }}$ without much effort: We obtain our quotients not in terms of generators and relations but get a clear view on the structure of these groups.

In view of Teicher's conjecture on virtual solvability of $\pi_{1}(\mathbb{A}-D)$, where $D$ is the branch curve of a generic projection from a surface $X$, we contribute the following result which shows that there are plenty of examples where $\pi_{1}\left(\mathbb{A}^{2}-D\right)$ is not virtually solvable.

Proposition 4.13, If $\pi_{1}(X)$ is not virtually solvable, then neither is $\pi_{1}\left(\mathbb{A}^{2}-D\right)$.

To state the precise results, we have to introduce some notation. We choose a generic line in $\mathbb{P}^{2}$ and denote its complement by $\mathbb{A}^{2}$. Then we consider the preimages of $\mathbb{A}^{2}$ on $X$ and $X_{\text {gal }}$ and obtain the following diagram:

$$
\begin{array}{ccccc}
X_{\text {gal }} & \rightarrow & X & \stackrel{f}{\rightarrow} & \mathbb{P}^{2} \\
\cup & & \cup & & \cup \\
X_{\text {gal }}^{\text {aff }} & \rightarrow & X^{\text {aff }} & \stackrel{f}{\rightarrow} & \mathbb{A}^{2} .
\end{array}
$$

For technical reasons ("monodromy at infinity") it is easier to compute the fundamental group $\pi_{1}\left(X_{\text {gal }}^{\text {aff }}\right)$ first.

Modulo certain relations, the group $\pi_{1}\left(X_{\text {gal }}^{\text {aff }}\right)$ depends only on $\pi_{1}\left(X^{\text {aff }}\right)$ and the degree $n$ of the generic projection.

Theorem 3.6. There exists a normal subgroup $C^{\mathrm{aff}}$ of $\pi_{1}\left(X_{\mathrm{gal}}^{\mathrm{aff}}\right)$ and an isomorphism

$$
\pi_{1}\left(X_{\text {gal }}^{\mathrm{aff}}\right) / C^{\mathrm{aff}} \cong \widetilde{\mathcal{K}}\left(\pi_{1}\left(X^{\mathrm{aff}}\right), n\right) .
$$


Here, $\widetilde{\mathcal{K}}\left(\pi_{1}\left(X^{\text {aff }}\right), n\right)$ is a purely group theoretical construction, which is an extension of $H_{2}\left(\pi_{1}\left(X^{\text {aff }}\right), \mathbb{Z}\right)$ by a certain subgroup of $\pi_{1}\left(X^{\text {aff }}\right)^{n}$.

The group $\pi_{1}\left(X_{\text {gal }}\right)$ is a quotient of $\pi_{1}\left(X_{\text {gal }}^{\text {aff }}\right)$ by a cyclic subgroup. The isomorphism of Theorem 3.6 is not well-behaved with respect to forming this quotient. In particular, if we denote by $C^{\text {proj }}$ the image of $C^{\text {aff }}$ in $\pi_{1}\left(X_{\text {gal }}\right)$, then $\pi_{1}\left(X_{\text {gal }}\right) / C^{\text {proj }}$ cannot be described in terms of $n=\operatorname{deg} f$ and $\pi_{1}(X)$ alone.

Theorem 4.5. There exists a cyclic group $Z$ and a central short exact sequence

$$
0 \rightarrow \frac{H_{2}\left(\pi_{1}\left(X^{\text {aff }}\right), \mathbb{Z}\right)}{Z} \rightarrow \pi_{1}\left(X_{\text {gal }}\right) / C^{\text {proj }} \rightarrow G \rightarrow 1,
$$

where $G$ is a group that is itself a central extension

$$
0 \rightarrow \operatorname{ker} \kappa_{n-1} \rightarrow G \rightarrow \mathcal{K}\left(\pi_{1}(X), n\right) \rightarrow 1 .
$$

Here, $\mathcal{K}\left(\pi_{1}(X), n\right)$ is a certain subgroup of $\pi_{1}(X)^{n}$. The group ker $\kappa_{n-1}$ is a subgroup of $K^{n-1}$, where $K$ is a cyclic group.

We show how to compute ker $\kappa_{n-1}$ and $H_{2}\left(\pi_{1}\left(X^{\text {aff }}\right), \mathbb{Z}\right)$ in practice in Section 5 , Proposition 4.11, Corollary 4.9 and Corollary 4.6 quoted above are applications of this theorem.

However, the main question that remains is the following

Question 3.4. Is $C^{\text {aff }}$ trivial for every generic projection of degree $n \geq 5$ ?

This is true in all known examples. However, since not many examples with non-trivial $\pi_{1}(X)$ have been computed, we do not dare to call it a conjecture. The assumption on the degree is necessary: A generic projection from the Veronese surface $V_{4}$ in $\mathbb{P}^{5}$, which has only degree 4 , leads to a nontrivial $C^{\text {aff }}$; cf. Remark 5.3 .

We now relate Question 3.4 to the computations of Moishezon, Teicher and others. To calculate $\pi_{1}\left(X_{\text {gal }}^{\text {aff }}\right)$, one first needs a presentation of the group $\pi_{1}\left(\mathbb{A}^{2}-D\right)$, where $D$ is the branch curve of the generic projection. Using degeneration techniques, one then shows that certain commutator and triple commutator relations hold between the standard generators of $\pi_{1}\left(\mathbb{A}^{2}-D\right)$; cf. [MoTe1, Proposition 2]. In our setup this amounts to proving that the group $C^{\text {aff }}$ is trivial. Hence answering Question 3.4 is a crucial step in the calculation of $\pi_{1}\left(X_{\text {gal }}^{\text {aff }}\right)$. Once we know that $C^{\text {aff }}$ is trivial, we can determine $\pi_{1}\left(X_{\text {gal }}\right)$, which already simplifies the existing computations greatly.

In any case, our results shed new light on $\pi_{1}\left(X_{\text {gal }}\right)$ and open new perspectives.

- Theorem 4.5 and its corollaries tell us that $\pi_{1}\left(X_{\text {gal }}\right) / C^{\text {proj }}$ is not too far away from $\pi_{1}(X)$. For example, if $X$ is simply connected, $\pi_{1}\left(X_{\text {gal }}\right) / C^{\text {proj }}$ does not contain interesting information on $X$. Even if this group is hard to determine, our structure results and Question 3.4 suggest that $\pi_{1}\left(X_{\text {gal }}\right)$ does not give rise to new invariants of $X$.

- The group $H_{2}\left(\pi_{1}\left(X^{\text {aff }}\right), \mathbb{Z}\right)$ maps to the commutator subgroup of $\pi_{1}\left(X_{\text {gal }}\right) /$ $C^{\text {proj }}$ (Theorem 4.5). Unfortunately, the known examples are too special so that this homological contribution has not yet appeared. Since it is still not known what kind of infinite groups can occur as fundamental groups of surfaces, one could use iterated Galois closures of generic projections and Corollary 4.7 to construct surfaces with nilpotent fundamental groups of arbitrary large nilpotency class. 
The article is organised as follows.

In Section 1 we review some facts about generic projections and their Galois closures.

In Section 2 we introduce two purely group theoretical constructions. Both depend on a group $G$ and a natural number $n \geq 3$. The first construction, called $\mathcal{K}(G, n)$, is a certain subgroup of $G^{n}$ and quite easy to calculate. The second one, called $\widetilde{\mathcal{K}}(G, n)$, is a central extension of $H_{2}(G, \mathbb{Z})$ by $\mathcal{K}(G, n)$ and quite difficult to determine in general. There is a surprising connection between $\widetilde{\mathcal{K}}(G, n)$ and the theory of central extensions whose meaning is unclear at the moment. We refer to Li2] for purely group theoretical properties of $\widetilde{\mathcal{K}}(G, n)$.

In Section 3 we consider the short exact sequence

$$
\text { (44) } 1 \rightarrow \pi_{1}\left(X_{\text {gal }}^{\text {aff }}\right) \rightarrow \pi_{1}\left(\mathbb{A}^{2}-D\right) /\left\langle\left\langle\Gamma_{i}{ }^{2}\right\rangle\right\rangle \rightarrow \mathfrak{S}_{n} \rightarrow 1 \text {, }
$$

which is also the starting point of the calculations of Moishezon and Teicher in MoTe1. Here, $D$ is the branch curve of the generic projection $f: X \rightarrow \mathbb{P}^{2}$, the $\Gamma_{i}$ 's are standard generators for $\pi_{1}\left(\mathbb{A}^{2}-D\right)$ and $\mathfrak{S}_{n}$ is the symmetric group on $n:=\operatorname{deg} f$ letters. We introduce a normal subgroup $C^{\text {aff }}$ of $\pi_{1}\left(\mathbb{A}^{2}-D\right)$. This group is trivial if certain commutator and triple commutator relations hold between the $\Gamma_{i}$ 's; cf. Definition 3.3. After taking the quotient of the short exact sequence (41) by $C^{\text {aff }}$, the resulting short exact sequence splits in a very nice way. Using the algorithm of Zariski and van Kampen and the results of Section 2, we obtain Theorem 3.6.

In Section 4 we determine the structure of $\pi_{1}\left(X_{\text {gal }}\right) / C^{\text {proj }}$ and give some applications.

In Section 5 we compute these quotients of $\pi_{1}\left(X_{\text {gal }}\right)$ and $\pi_{1}\left(X_{\text {gal }}^{\text {aff }}\right)$ for all examples that are known to the author. In particular, we demonstrate how to avoid combinatorial group theory once our theorems are at our disposal.

\section{Generic projections and their Galois closures}

In this section we recall some general facts about generic projections and their Galois closures. Most of these results are well known. We follow [Fa, Section 2].

Definition 1.1. Let $X$ be a smooth complex projective surface. A line bundle $\mathcal{L}$ on $X$ is called sufficiently ample if

(1) $\mathcal{L}$ is an ample line bundle with self-intersection number at least 5.

(2) For every closed point $x \in X$ the global sections of $\mathcal{L}$ generate the fibre

$$
\mathcal{L}_{x} / \mathfrak{m}_{x}{ }^{4} \mathcal{L}
$$

(3) For any pair $\{x, y\}$ of distinct closed points of $X$ the global sections of $\mathcal{L}$ generate the direct sum

$$
\mathcal{L}_{x} / \mathfrak{m}_{x}{ }^{3} \mathcal{L} \oplus \mathcal{L}_{y} / \mathfrak{m}_{y}{ }^{3} \mathcal{L}
$$

(4) For any triple $\{x, y, z\}$ of distinct closed points the global sections of $\mathcal{L}$ generate the direct sum

$$
\mathcal{L}_{x} / \mathfrak{m}_{x}{ }^{2} \mathcal{L} \oplus \mathcal{L}_{y} / \mathfrak{m}_{y}{ }^{2} \mathcal{L} \oplus \mathcal{L}_{z} / \mathfrak{m}_{z}{ }^{2} \mathcal{L} .
$$

The following remark was already made in $\mathrm{Fa}$.

Remark 1.2. The tensor product of five very ample line bundles is sufficiently ample. 
Definition 1.3. Let $\mathcal{L}$ be a sufficiently ample line bundle on the surface $X$. We call a three-dimensional linear subspace $E \subseteq H^{0}(X, \mathcal{L})$ generic if

(1) $E$ generates $\mathcal{L}$, i.e., the associated rational map is in fact a finite morphism

$$
f=f_{E}: X \rightarrow \mathbb{P}(E) \cong \mathbb{P}^{2}
$$

of degree $n$ equal to the self-intersection number of $\mathcal{L}$.

(2) The ramification locus $R$ of $f_{E}$ is a smooth and ample curve on $X$. The ramification index at a generic point is 2 .

(3) The branch locus $D$ of $f_{E}$ is a curve on $\mathbb{P}^{2}$ with at worst cusps and nodes as singularities.

(4) The restriction $\left.f_{E}\right|_{R}: R \rightarrow D$ is birational.

We call the finite morphism $f_{E}$ associated to a generic subspace of $H^{0}(X, \mathcal{L})$ a generic projection.

Let $\mathbb{G}(k, V)$ be the Grassmannian parametrising $k$-dimensional linear subspaces of the vector space $V$. To justify the name in the previous definition we have

Proposition 1.4. Let $\mathcal{L}$ be a sufficiently ample line bundle on the surface $X$. Then there exists a dense and open subset $G^{\prime}$ of $\mathbb{G}\left(3, H^{0}(X, \mathcal{L})\right)$ such that all $E \in G^{\prime}$ are generic in the sense of Definition 1.3 .

Proof. A proof is given in [Fa, Proposition 1]. For the conclusions there it is necessary that the line bundle $\mathcal{K}_{X} \otimes \mathcal{L}^{\otimes 3}$ is ample. In our setup, this follows from the assumptions on the self-intersection number of $\mathcal{L}$ and Reider's theorem Re.

Let $f: X \rightarrow P$ be a finite morphism between normal complex surfaces. Let $L$ be the Galois closure of the field extension of function fields $K(P) \hookrightarrow K(X)$ induced by $f$. Let $X_{\text {gal }}$ be the normalisation of $X$ or, equivalently, of $P$ in $L$.

Definition 1.5. Given a finite morphism $f: X \rightarrow P$ of normal complex surfaces we call the normal surface $X_{\text {gal }}$, together with the induced morphism $f_{\text {gal }}: X_{\text {gal }} \rightarrow P$, the Galois closure of $f$.

The Galois closure of a field extension $K / k$ of degree $n$ is isomorphic to a quotient of the $n$-fold tensor product of $K$ with itself over $k$. Similarly (cf. SGA1, Exposé $\mathrm{V} .4 \mathrm{~g}]$ ), the Galois closure of a generic projection is isomorphic to the closure of a certain subset of $X^{n}$ :

$$
X_{\text {gal }} \cong \overline{\left\{\left(x_{1}, \ldots, x_{n}\right) \mid x_{i} \neq x_{j}, f\left(x_{i}\right)=f\left(x_{j}\right), \forall i \neq j\right\}} \subseteq X^{n} .
$$

For Galois closures of generic projections we have the following proposition, which is proved for our setup in [Fa, Proposition 1].

Proposition 1.6. Let $\mathcal{L}$ be a sufficiently ample line bundle on the surface $X$ with self-intersection $n$. Then there exists an open and dense subset $G$ of $\mathbb{G}\left(3, H^{0}(X, \mathcal{L})\right)$ such that for all $E \in G$

(1) The map $f_{E}$ associated to $E$ is a generic projection of degree $n$.

(2) The Galois closure $X_{\text {gal }}$ of $f_{E}$ is a smooth projective surface.

(3) The Galois group $\operatorname{Gal}\left(K\left(X_{\text {gal }}\right) / K\left(\mathbb{P}^{2}\right)\right)$ is the symmetric group $\mathfrak{S}_{n}$.

(4) The branch curve $D$ of $f_{E}$ is an irreducible curve in $\mathbb{P}^{2}$.

Definition 1.7. We call a map $f_{E}$ as in Proposition 1.6 a good generic projection from $X$.

Explicit formulae for the degree of the branch curve $D$ and the number of cusps and nodes on $D$ can be found in [Fa, Section 4] or [MoTe1, Chapter 0]. 


\section{TWO GROUP THEORETICAL CONSTRUCTIONS}

This section contains the group theoretical heart of this article. Given a group $G$ and a natural number $n \geq 3$, we introduce the two associated groups $\mathcal{K}(G, n)$ and $\widetilde{\mathcal{K}}(G, n)$. The second construction is related to the theory of central extensions. We refer to [Li2] for details, further properties and examples.

The first construction. We let $G$ be a group and $n \geq 3$ be a natural number. We denote by $G^{\mathrm{ab}}:=G /[G, G]$ the Abelianisation of $G$. Then we define a map

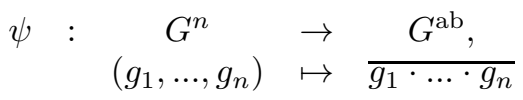

which is a homomorphism since $G^{\text {ab }}$ is an Abelian group.

Definition 2.1. For a group $G$ and a natural number $n \geq 3$, we define $\mathcal{K}(G, n)$ to be the kernel of the homomorphism $\psi: G^{n} \rightarrow G^{\mathrm{ab}}$.

For Abelian groups it is particularly easy to determine $\mathcal{K}(G, n)$.

Proposition 2.2. There exists a natural isomorphism $\mathcal{K}(G, n)^{\mathrm{ab}} \cong \mathcal{K}\left(G^{\mathrm{ab}}, n\right)$. If $G$ is Abelian, then $\mathcal{K}(G, n) \cong G^{n-1}$. This isomorphism is not canonical.

Proof. The surjection $G \rightarrow G^{\mathrm{ab}}$ and the universal property of Abelianisation imply that there is a natural surjective homomorphism $\mathcal{K}(G, n)^{\text {ab }} \rightarrow \mathcal{K}\left(G^{\mathrm{ab}}, n\right)$. An element of the kernel $\mathcal{K}(G, n) \rightarrow \mathcal{K}\left(G^{\mathrm{ab}}, n\right)$ is also an element of the kernel $G^{n} \rightarrow\left(G^{\mathrm{ab}}\right)^{n}$, which is $[G, G]^{n}$. Using $n \geq 3$ we may write

$$
\left(\left[h_{1}, h_{2}\right], 1, \ldots, 1\right)=\left[\left(h_{1}, h_{1}^{-1}, 1, \ldots, 1\right),\left(h_{2}, 1, h_{2}^{-1}, \ldots, 1\right)\right] .
$$

Thus $[G, G]^{n}$ is not only a subgroup of $\mathcal{K}(G, n)$ but also lies inside the commutator subgroup of $\mathcal{K}(G, n)$. Hence the kernel $\mathcal{K}(G, n) \rightarrow \mathcal{K}\left(G^{\text {ab }}, n\right)$ is the commutator subgroup of $\mathcal{K}(G, n)$, and we are done.

Let $G$ be Abelian. Then the map

$$
\begin{array}{ccc}
G^{n-1} & \rightarrow & G^{n}, \\
\left(g_{1}, \ldots, g_{n-1}\right) & \mapsto & \left(g_{1}, \ldots, g_{n-1},\left(g_{1} \cdot \ldots \cdot g_{n-1}\right)^{-1}\right)
\end{array}
$$

defines a homomorphism. It is injective with image $\mathcal{K}(G, n)$.

The symmetric group $\mathfrak{S}_{n}$ acts on $G^{n}$ by permutation of the $n$ factors. This action induces an action $\varphi$ of $\mathfrak{S}_{n}$ on $\mathcal{K}(G, n)$. With respect to this action we form the semidirect product

$$
\mathcal{E}(G, n):=\mathcal{K}(G, n) \rtimes_{\varphi} \mathfrak{S}_{n}
$$

For $\vec{g} \in \mathcal{K}(G, n)$ and $\sigma \in \mathfrak{S}_{n}$ we will write $[\vec{g}, \sigma]$ for $\vec{g} \cdot \varphi(\sigma)\left(\vec{g}^{-1}\right)$.

The main construction. Again, we let $G$ be a group and $n \geq 3$ be a natural number. We choose a presentation $G \cong F / N$, where $F$ is a free group. Then $\mathcal{K}(N, n)$ is a subgroup of $\mathcal{K}(F, n)$, which is a subgroup of $F^{n}$.

We denote by $\langle\langle\mathcal{K}(N, n)\rangle\rangle$ the subgroup normally generated by $\mathcal{K}(N, n)$ inside $F^{n}$. Using formula (1), it is not hard to verify that the group $\langle\langle\mathcal{K}(N, n)\rangle\rangle$ is equal to the subgroup normally generated by $\mathcal{K}(N, n)$ inside $\mathcal{K}(F, n)$.

Definition 2.3. Let $G$ be a group and $n \geq 3$ be a natural number. We define

$$
\widetilde{\mathcal{K}}(G, n):=\mathcal{K}(F, n) /\langle\langle\mathcal{K}(N, n)\rangle\rangle .
$$


Theorem 2.4. The group $\widetilde{\mathcal{K}}(G, n)$ does not depend upon the choice of a presentation. There exists a central short exact sequence

$$
0 \rightarrow H_{2}(G, \mathbb{Z}) \rightarrow \widetilde{\mathcal{K}}(G, n) \rightarrow \mathcal{K}(G, n) \rightarrow 1,
$$

where $H_{2}(G, \mathbb{Z})$ denotes the second group homology with integer coefficients. The group $\mathrm{H}_{2}(G, \mathbb{Z})$ lies inside the commutator subgroup of $\widetilde{\mathcal{K}}(G, n)$.

Proof. We choose a presentation $G \cong F / N$ and abbreviate the normal closure of $\mathcal{K}(N, n)$ in $F^{n}$ by $R$. Also, we will simply write $H_{2}(G)$ for $H_{2}(G, \mathbb{Z})$.

First, we prove the short exact sequence (2): We denote by $\pi$ the projection of $F^{n}$ onto its last $n-1$ factors. By abuse of notation we will denote its restriction to $\mathcal{K}(F, n)$ also by $\pi$. Then we have a short exact sequence

$$
1 \rightarrow[F, F] \rightarrow \mathcal{K}(F, n) \stackrel{\pi}{\rightarrow} F^{n-1} \rightarrow 1 .
$$

An easy computation with commutators shows that $R \cap \operatorname{ker} \pi=[F, N]$. Via $\pi$ we obtain the following diagram with exact rows and columns:

$$
\begin{aligned}
& 1 \rightarrow[F, N] \quad \rightarrow \quad R \quad \rightarrow \quad N^{n-1} \rightarrow 1
\end{aligned}
$$

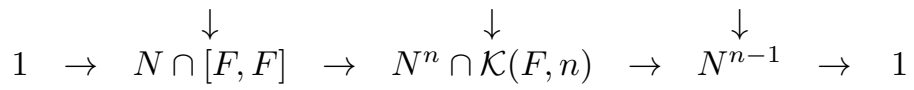

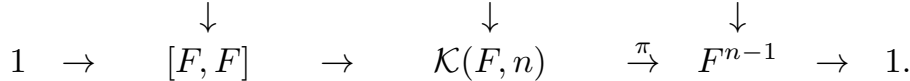

Taking quotients of successive rows we exhibit the group $\mathcal{K}(F, n) / R$ as an extension of $(N \cap[F, F]) /[F, N]$ by $\mathcal{K}(F, n) /\left(N^{n} \cap \mathcal{K}(F, n)\right)$. The latter group is isomorphic to $\mathcal{K}(G, n)$. By Hopf's theorem $\mathrm{Br}$, Theorem II.5.3] the group $(N \cap[F, F]) /[F, N]$ is isomorphic to $H_{2}(G)$. Hence we obtain an extension

$$
1 \rightarrow H_{2}(G) \rightarrow \mathcal{K}(F, n) / R \rightarrow \mathcal{K}(G, n) \rightarrow 1 .
$$

Now we will show that this extension is central: Every element of $H_{2}(G)$ can be lifted to an element $x \in \mathcal{K}(F, n)$ of the form $\vec{x}:=(x, 1, \ldots, 1)$ with $x \in N \cap[F, F]$. For $\vec{y}:=\left(y_{1}, \ldots, y_{n}\right) \in \mathcal{K}(F, n)$ we compute

$$
\vec{y} \vec{x} \vec{y}^{-1}=(\underbrace{\left[y_{1}, x\right]}_{\in[F, N]}, 1, \ldots, 1) \cdot(x, 1, \ldots, 1) \equiv \vec{x} \quad \bmod [F, N] .
$$

Hence $H_{2}(G)$ lies inside the centre of $\mathcal{K}(F, n) / R$.

We now prove that $\widetilde{\mathcal{K}}(G, n)$ is well defined: Let $\alpha: F / N \cong F^{\prime} / N^{\prime}$ be another presentation for $G$. We may lift this isomorphism to a map $\varphi: F \rightarrow F^{\prime}$. Then $\varphi$ maps $N$ to $N^{\prime}$ and hence $\mathcal{K}(N, n)$ to $\mathcal{K}\left(N^{\prime}, n\right)$. Let $R^{\prime}$ be the normal closure of $\mathcal{K}\left(N^{\prime}, n\right)$ inside $F^{\prime n}$. Then $\varphi$ induces a homomorphism

$$
\bar{\varphi}: \mathcal{K}(F, n) / R \rightarrow \mathcal{K}\left(F^{\prime}, n\right) / R^{\prime} .
$$

Let $\varphi^{\prime}$ be another map lifting $\alpha$ to a homomorphism from $F$ to $F^{\prime}$.

Elements of the form $\left(f, f^{-1}, 1, \ldots, 1\right)$ generate $\mathcal{K}(F, n)$. Hence it is enough to compare the induced maps on these elements. For $f \in F$ there exists $n_{f}^{\prime} \in N^{\prime}$ such that $\varphi(f)=\varphi^{\prime}(f) n_{f}^{\prime}$. Hence

$$
\begin{aligned}
\varphi\left(\left(f, f^{-1}, \ldots\right)\right) & =\left(\varphi^{\prime}(f) n_{f}^{\prime}, n_{f}^{\prime-1} \varphi^{\prime}(f)^{-1}, \ldots\right) \\
& =\varphi^{\prime}\left(\left(f, f^{-1}, \ldots\right)\right) \underbrace{\left(n_{f}^{\prime}, \varphi^{\prime}(f) n_{f}^{\prime-1} \varphi^{\prime}(f)^{-1}, \ldots\right)}_{\in R^{\prime}} .
\end{aligned}
$$

Hence the induced maps coincide. 
In particular, if we choose $F=F^{\prime}$ and $N=N^{\prime}$ with $\alpha$ and $\varphi$ the identity, then every other lift $\varphi^{\prime}$ of the identity induces the identity on $\mathcal{K}(F, n) / R$.

Coming back to the general case, we let $F / N$ and $F^{\prime} / N^{\prime}$ again be two presentations of $G$ and let $\alpha$ be an isomorphism between them. Then $\alpha$ and $\alpha^{-1}$ induce maps between $\mathcal{K}(F, n) / R$ and $\mathcal{K}\left(F^{\prime}, n\right) / R^{\prime}$ such that the composites of these induced maps are the identity by the previous paragraph. Hence $\alpha$ induces an isomorphism from $\mathcal{K}(F, n) / R$ to $\mathcal{K}\left(F^{\prime}, n\right) / R^{\prime}$. Thus, $\widetilde{\mathcal{K}}(G, n)$ is well defined.

Taking the quotient of the top row by the bottom row of (3) we obtain an exact sequence

$$
1 \rightarrow[F, F] /[F, N] \rightarrow \mathcal{K}(F, n) / R \rightarrow G^{n-1} \rightarrow 1 .
$$

The inclusion of $H_{2}(G)$ into $\mathcal{K}(F, n) / R$ factors over $[F, F] /[F, N]$. The group $[F, F]$ lies inside the commutator subgroup of $\mathcal{K}(F, n)$; cf. formula (11). Hence $H_{2}(G)$ lies inside the commutator subgroup of $\mathcal{K}(F, n) / R$.

Whenever the symmetric group $\mathfrak{S}_{n}$ acts on a group $X$ via some homomorphism $\varphi: \mathfrak{S}_{n} \rightarrow \operatorname{Aut}(X)$, we may form the semidirect product $X \rtimes \mathfrak{S}_{n}$ with respect to this action. For $x \in X$ and $\sigma \in \mathfrak{S}_{n}$ the commutator $[x, \sigma]$ in $X \rtimes \mathfrak{S}_{n}$ is equal to $x \sigma x^{-1} \sigma^{-1}=x \cdot \varphi(\sigma)\left(x^{-1}\right)$ by definition of the semidirect product. For a subgroup $S$ of $\mathfrak{S}_{n}$ we define $[X, S]$ to be the subgroup of $X \rtimes \mathfrak{S}_{n}$ generated by all elements $[x, s]$ with $x \in X$ and $s \in S$. It is easy to see that $[X, S]$ is a normal subgroup of $X$.

We already mentioned that there is an action of $\mathfrak{S}_{n}$ on $\mathcal{K}(G, n)$ for every group $G$. In the following we embed $\mathfrak{S}_{n-1}$ into $\mathfrak{S}_{n}$ as the subgroup of those permutations that fix the first letter.

Corollary 2.5. There exists a $\mathfrak{S}_{n}$-action on $\widetilde{\mathcal{K}}(G, n)$ such that the homomorphism from $\widetilde{\mathcal{K}}(G, n)$ onto $\mathcal{K}(G, n)$ becomes equivariant. There exists an isomorphism

$$
\widetilde{\mathcal{K}}(G, n) /\left[\widetilde{\mathcal{K}}(G, n), \mathfrak{S}_{n-1}\right] \cong G .
$$

Proof. Given a presentation $G \cong F / N$ it is easy to check that the $\mathfrak{S}_{n}$-action on $\mathcal{K}(F, n)$ induces an action on $\widetilde{\mathcal{K}}(G, n)$ that does not depend on the choice of the presentation.

We view $\mathcal{K}(F, n)$ as a subgroup of $F^{n}$. We let $\mathfrak{S}_{n-1}$ be the subgroup of $\mathfrak{S}_{n}$ of those permutations that fix the first letter. The group $\left[\mathcal{K}(F, n), \mathfrak{S}_{n-1}\right]$ is a normal subgroup of $\mathcal{K}(F, n)$ consisting of those elements with a trivial entry in the first coordinate. Hence

$$
\mathcal{K}(F, n) /\left[\mathcal{K}(F, n), \mathfrak{S}_{n-1}\right] \cong F .
$$

Of course, the same holds true if we replace $F$ by $N$. We have isomorphisms

$$
\frac{\widetilde{\mathcal{K}}(G, n)}{\left[\widetilde{\mathcal{K}}(G, n), \mathfrak{S}_{n-1}\right]} \cong \frac{\mathcal{K}(F, n)}{\left[\mathcal{K}(F, n), \mathfrak{S}_{n-1}\right]} / \frac{\mathcal{K}(N, n)}{\left[\mathcal{K}(N, n), \mathfrak{S}_{n-1}\right]} \cong F / N \cong G .
$$

This proves our assertion.

Corollary 2.6. If $G$ is cyclic, then $\widetilde{\mathcal{K}}(G, n) \cong G^{n-1}$. This isomorphism is not canonical. 
Proof. If $G$ is cyclic, then $H_{2}(G, \mathbb{Z})$ vanishes. Hence $\widetilde{\mathcal{K}}(G, n)$ is isomorphic to $\mathcal{K}(G, n)$, which is isomorphic to $G^{n-1}$ by Proposition 2.2 .

Corollary 2.7. There exist isomorphisms $\widetilde{\mathcal{K}}(G, n)^{\mathrm{ab}} \cong \mathcal{K}(G, n)^{\mathrm{ab}} \cong\left(G^{\mathrm{ab}}\right)^{n-1}$.

Proof. The first isomorphism follows from the fact that $H_{2}(G, \mathbb{Z})$ lies inside the commutator subgroup of $\widetilde{\mathcal{K}}(G, n)$. The second isomorphism follows from Proposition 2.2 ,

A presentation of $\mathcal{E}(F, n)$ and affine subgroups. Let $F$ be a free group. Then $\mathcal{K}(F, n)$ is a subgroup of $F^{n}$. We recall that there exists an action $\varphi$ of the symmetric group $\mathfrak{S}_{n}$ on $\mathcal{K}(F, n)$. For $\vec{f} \in \mathcal{K}(F, n)$ and $\sigma \in \mathfrak{S}_{n}$, we refer to $\varphi(\sigma)(\vec{f})$ as the element that is $\sigma$-conjugate to $\vec{f}$.

Definition 2.8. Let $F$ be a free group. A normal subgroup of $\mathcal{E}(F, n)$ is called an affine subgroup if it is normally generated by elements of the form $\left(r, r^{-1}, 1, \ldots, 1\right)$ and their $\mathfrak{S}_{n}$-conjugates.

Clearly, affine subgroups of $\mathcal{K}(F, n)$ are $\mathfrak{S}_{n}$-invariant. The quotient of $\mathcal{K}(F, n)$ by an affine subgroup has a particularly nice structure:

Theorem 2.9. Let $F$ be a free group and let $R$ be an affine subgroup of $\mathcal{K}(F, n)$. Let $p: \mathcal{K}(F, n) \rightarrow F$ be the projection onto the first factor. We define $N:=p(R)$. Then there exists an isomorphism

$$
\mathcal{K}(F, n) / R \cong \widetilde{\mathcal{K}}(F / N, n) .
$$

In particular, this quotient is completely determined by $F / N$ and $n$.

Proof. As $p$ is surjective, $N=p(R)$ is a normal subgroup of $F$. Using $n \geq 3$ and formula (1) it is easy to see that $\mathcal{K}(N, n)$ is contained in $R$. As $R$ is a normal subgroup of $\mathcal{K}(F, n)$, the normal closure $\langle\langle\mathcal{K}(N, n)\rangle\rangle$ is also contained in $R$.

Conversely, $R$ is normally generated by elements of the form $\left(r, r^{-1}, \ldots\right)$ and their $\mathfrak{S}_{n}$-conjugates. But if $\left(r, r^{-1}, 1 \ldots\right)$ lies in $R$, then $r$ lies in $N$. We conclude that $R$ is contained in $\langle\langle\mathcal{K}(N, n)\rangle\rangle$. Thus $R$ is equal to $\langle\langle\mathcal{K}(N, n)\rangle\rangle$, and so $\mathcal{K}(F, n) / R$ is isomorphic to $\widetilde{\mathcal{K}}(F / N, n)$.

Let $F_{d-1}$ be the free group of rank $d-1$. We finish this section by giving a presentation of $\mathcal{E}\left(F_{d-1}, n\right)$ : Let $S_{d}$ be the free group of rank $d$ with basis $s_{i}$, $i=1, \ldots, d$. We form the free product of the symmetric group $S_{d}$ with $\mathfrak{S}_{n}$. We define

$$
\begin{array}{rlrl}
\psi \quad: \quad S_{d} * \mathfrak{S}_{n} & \rightarrow \mathfrak{S}_{n}, & \\
s_{i} & \mapsto & (12) & i=1, \ldots, d, \\
\sigma & \mapsto & \sigma & \forall \sigma \in \mathfrak{S}_{n} .
\end{array}
$$

We denote by $[a, b]:=a b a^{-1} b^{-1}$ and $\langle a, b\rangle:=a b a b^{-1} a^{-1} b^{-1}$ commutators and triple commutators, respectively. Then we let $R_{d, n}$ be the subgroup of $S_{d} * \mathfrak{S}_{n}$ normally generated by the elements

$$
\begin{array}{ll}
s_{i}{ }^{2} & \text { for } i=1, \ldots, d, \\
s_{1} \cdot(12)^{-1} & \text { i.e., we identify } s_{1} \text { with }(12), \\
{\left[s_{i}, \tau\right]} & \text { for transpositions } \tau \in \mathfrak{S}_{n} \text { disjoint from }(12), \\
\left\langle s_{i}, \tau\right\rangle & \text { for transpositions } \tau \in \mathfrak{S}_{n} \text { having one index } \\
& \text { in common with }(12), \\
{\left[\sigma s_{i} \sigma^{-1}, s_{j}\right]} & \text { if } \psi\left(\sigma s_{i} \sigma^{-1}\right) \text { and } \psi\left(s_{j}\right) \text { are disjoint, } \\
\left\langle\sigma s_{i} \sigma^{-1}, s_{j}\right\rangle & \text { if } \psi\left(\sigma s_{i} \sigma^{-1}\right) \text { and } \psi\left(s_{j}\right) \text { have one index in common. }
\end{array}
$$


Definition 2.10. We define $\mathcal{S}_{n}(d):=\left(S_{d} * \mathfrak{S}_{n}\right) / R_{n, d}$.

It is easy to see that the homomorphism $\psi$ factors over the quotient by $R_{n, d}$. By abuse of notation we will again call $\psi$ the induced map on $\mathcal{S}_{n}(d)$. We may think of $\mathcal{S}_{n}(d)$ as a symmetric group with $d$ "layers".

Let $F_{d-1}$ be the free group of rank $d-1$ with basis $f_{2}, \ldots, f_{d}$ (mind the numbering!). The group $\mathfrak{S}_{n}$ acts on $\left(F_{d-1}\right)^{n}$ by permuting the factors. We denote the corresponding semidirect product by $\left(F_{d-1}\right)^{n} \rtimes \mathfrak{S}_{n}$. It is easy to check that the map

$$
\begin{array}{rlrcrl}
\varphi: \mathcal{S}_{n}(d) & \rightarrow & \left(F_{d-1}\right)^{n} \rtimes \mathfrak{S}_{n}, & & \\
s_{1} & \mapsto & (12), & \\
s_{i} & \mapsto & \left(f_{i}, f_{i}{ }^{-1}, 1, \ldots, 1\right) \cdot(12) & i=2, \ldots, d, \\
\sigma & \mapsto & \sigma & & \forall \sigma \in \mathfrak{S}_{n}
\end{array}
$$

defines a homomorphism of groups. Its image lies inside $\mathcal{E}\left(F_{d-1}, n\right)$. Moreover, this latter group is generated by the $\mathfrak{S}_{n}$-conjugates of the elements $\left(f_{i}, f_{i}{ }^{-1}, 1, \ldots, 1\right)$. Hence $\varphi$ maps surjectively onto $\mathcal{E}\left(F_{d-1}, n\right)$. But even more is true.

Theorem 2.11. For $n \geq 5$ the map $\varphi$ defines an isomorphism

$$
\mathcal{S}_{n}(d) \cong \mathcal{E}\left(F_{d-1}, n\right) .
$$

In particular, $\mathcal{S}_{n}(d)$ defines a presentation of $\mathcal{E}\left(F_{d-1}, n\right)$.

Proof. Our original proof applied a Reidemeister-Schreier rewriting process to the kernel $\operatorname{ker}(\psi)$ of $\mathcal{S}_{n}(d)$. We obtained an infinite set of relations and checked that all of them follow from relations in $\left(F_{d-1}\right)^{n}$. This then implies that $\varphi$ is injective. The computations are straightforward, but quite lengthy.

In the meantime, the article [RTV] appeared independently from our work. So instead of giving our proof, we refer to [RTV, Theorem 5.7] instead.

\section{The fundamental group of the affine Piece}

Let $f: X \rightarrow \mathbb{P}^{2}$ be a good generic projection of degree $n$ given by a sufficiently ample line bundle as defined in Section [1. We denote by $f_{\text {gal }}: X_{\text {gal }} \rightarrow \mathbb{P}^{2}$ its Galois closure. We choose a generic line $\tilde{\ell}$ in $\mathbb{P}^{2}$, i.e., a line that intersects the branch curve $D$ of $f$ in $\mathbb{P}^{2}$ transversely.

We define $X^{\text {aff }}:=X-f^{-1}(\tilde{\ell})$ and $X_{\text {gal }}^{\text {aff }}:=X_{\text {gal }}-f_{\text {gal }}{ }^{-1}(\tilde{\ell})$. Then we obtain the following morphisms:

$$
\begin{array}{ccccc}
X_{\text {gal }} & \rightarrow & X & \stackrel{f}{\rightarrow} & \mathbb{P}^{2} \\
\cup & & \cup & & \cup \\
X_{\text {gal }}^{\text {aff }} & \rightarrow & X^{\text {aff }} & \stackrel{f}{\rightarrow} & \mathbb{A}^{2} .
\end{array}
$$

We choose a second line $\ell$ in $\mathbb{A}^{2}:=\mathbb{P}^{2}-\tilde{\ell}$ that intersects $D$ and $\tilde{\ell}$ transversely. Then we choose a system of simple loops $\Gamma_{i}$ around the intersection points $\ell \cap D$ inside $\ell-D$. There are $d:=\operatorname{deg} D$ such simple loops. The inclusion map of topological spaces induces a surjective homomorphism

$$
\pi_{1}(\ell-(D \cap \ell)) \rightarrow \pi_{1}\left(\mathbb{A}^{2}-D\right) .
$$

The fundamental group on the left is a free group of rank $d$ with basis $\left\{\Gamma_{i}\right\}, i=$ $1, \ldots, d$.

By definition of a generic projection (Definition 1.3), the branch curve $D$ has at worst cusps and nodes as singularities. 
Lemma 3.1. The simple loops $\Gamma_{i}, i=1, \ldots, \operatorname{deg} D$, generate $\pi_{1}\left(\mathbb{A}^{2}-D\right)$. All relations in this group follow from relations of the type

$$
\gamma \Gamma_{i} \gamma^{-1} \cdot \Gamma_{j}^{-1},\left[\gamma \Gamma_{i} \gamma^{-1}, \Gamma_{j}\right],\left\langle\gamma \Gamma_{i} \gamma^{-1}, \Gamma_{j}\right\rangle
$$

for elements $\gamma \in \pi_{1}\left(\mathbb{A}^{2}-D\right)$.

Proof. This follows from the theorem of Zariski and van Kampen on fundamental groups of complements. A modern proof is given in [Ch, Théorème 4.1]. The relations occurring if the curve has at worst cusps and nodes are determined in [Ch, Partie 6.2].

We lift loops around $x_{0}$ to points of $f^{-1}\left(x_{0}\right)$ and obtain paths in $X^{\text {aff }}$. Clearly, the endpoints of these paths are again points of that fibre. Thus every loop around $x_{0}$ defines a permutation of $f^{-1}\left(x_{0}\right)$, which is a set with $n$ elements. So there exists a homomorphism $\psi$ from $\pi_{1}\left(\mathbb{A}^{2}-D\right)$ to the symmetric group $\mathfrak{S}_{n}$.

The Galois group of the extension of the function fields of $X_{\text {gal }}$ over $\mathbb{P}^{2}$ is isomorphic to $\mathfrak{S}_{n}$ by Proposition 1.6. It follows that

$$
\psi: \pi_{1}\left(\mathbb{A}^{2}-D, x_{0}\right) \rightarrow \mathfrak{S}_{n}
$$

is surjective. Clearly, the kernel of $\psi$ is isomorphic to $\pi_{1}\left(X_{\text {gal }}^{\text {aff }}-f_{\text {gal }}{ }^{-1}(D)\right)$.

Over a smooth point of $D$ there are $n-1$ points of $X$. One of them belongs to the ramification divisor of $f$ and has ramification index equal to 2 , whereas the other points are unramified; cf. Definition 1.3. It follows that the $\Gamma_{i}$ 's map to transpositions in $\mathfrak{S}_{n}$. In particular, the subgroup normally generated by the $\Gamma_{i}^{2}$, s inside $\pi_{1}\left(\mathbb{A}^{2}-D\right)$ lies in ker $\psi$.

The following lemma extends a result of [MoTe1, Chapter 0.3].

Lemma 3.2. Let $G_{n}$ be the subgroup normally generated by the $\Gamma_{i}^{2}$ 's in $\pi_{1}\left(\mathbb{A}^{2}-D\right)$. We view $\mathfrak{S}_{n-1}$ as a subgroup of $\mathfrak{S}_{n}$ fixing one of the letters. Define $G_{n-1}$ to be the following normal subgroup of $\psi^{-1}\left(\mathfrak{S}_{n-1}\right)$ :

$$
G_{n-1}:=\left\langle\left\langle G_{n}, \gamma \Gamma_{i} \gamma^{-1} \mid \psi\left(\gamma \Gamma_{i} \gamma^{-1}\right) \in \mathfrak{S}_{n-1}, \gamma \in \pi_{1}\left(\mathbb{A}^{2}-D\right)\right\rangle\right\rangle .
$$

Then there exist isomorphisms

$$
\begin{array}{ll}
\operatorname{ker} \psi / G_{n} & \cong \pi_{1}\left(X_{\text {gal }}^{\text {aff }}\right), \\
\psi^{-1}\left(\mathfrak{S}_{n-1}\right) / G_{n-1} & \cong \pi_{1}\left(X^{\text {aff }}\right) .
\end{array}
$$

Proof. It is shown in [MoTe1, Chapter 0.3] that the $\Gamma_{i}^{2}$ lift to loops around the ramification divisor $f_{\text {gal }}^{-1}(D)$ and that these loops generate the kernel of the surjective homomorphism from ker $\psi \cong \pi_{1}\left(X_{\text {gal }}^{\text {aff }}-f_{\text {gal }}{ }^{-1}(D)\right)$ onto $\pi_{1}\left(X_{\text {gal }}^{\text {aff }}\right)$.

The same arguments prove that $G_{n-1}$ is the kernel of the surjective homomorphism from $\psi^{-1}\left(\mathfrak{S}_{n-1}\right) \cong \pi_{1}\left(X^{\text {aff }}-f^{-1}(D)\right)$ onto $\pi_{1}\left(X^{\text {aff }}\right)$.

The isomorphism $\operatorname{ker} \psi / G_{n} \cong \pi_{1}\left(X_{\text {gal }}^{\text {aff }}\right)$ shows that there is a short exact sequence

$$
1 \rightarrow \pi_{1}\left(X_{\text {gal }}^{\mathrm{aff}}\right) \rightarrow \pi_{1}\left(\mathbb{A}^{2}-D, x_{0}\right) /\left\langle\left\langle\Gamma_{i}{ }^{2}\right\rangle\right\rangle \stackrel{\psi}{\rightarrow} \mathfrak{S}_{n} \rightarrow 1 .
$$

It is the starting point of the computations in [MoTe1, Chapter 0.3]. In fact, they prove that this short exact sequence splits. After that, they use a ReidemeisterSchreier rewriting process to obtain a presentation of $\pi_{1}\left(X_{\mathrm{gal}}^{\mathrm{aff}}\right)$. 
Definition 3.3. We define $C^{\text {aff }}$ to be the subgroup normally generated by the following elements inside $\pi_{1}\left(\mathbb{A}^{2}-D, x_{0}\right)$ :

$\left[\gamma \Gamma_{i} \gamma, \Gamma_{j}^{-1}\right] \quad$ if $\psi\left(\gamma \Gamma_{i} \gamma^{-1}\right)$ and $\psi\left(\Gamma_{j}\right)$ are disjoint transpositions,

$\left\langle\gamma \Gamma_{i} \gamma, \Gamma_{j}^{-1}\right\rangle$ if $\psi\left(\gamma \Gamma_{i} \gamma^{-1}\right)$ and $\psi\left(\Gamma_{j}\right)$ have precisely one letter in common,

where $\gamma$ runs through $\pi_{1}\left(\mathbb{A}^{2}-D, x_{0}\right)$.

Question 3.4. Is the group $C^{\text {aff }}$ trivial for every good generic projection?

This is the main problem that we cannot solve. However, we will see in Section 5 that the answer to this question is positive in all known examples up to now. So the reader should bear in mind that we hope for $C^{\text {aff }}$ to be trivial.

Since $C^{\text {aff }}$ lies inside ker $\psi$, the short exact sequence (4) induces a short exact sequence

$$
1 \rightarrow \pi_{1}\left(X_{\text {gal }}^{\text {aff }}\right) / C^{\text {aff }} \rightarrow \frac{\pi_{1}\left(\mathbb{A}^{2}-D, x_{0}\right)}{\left\langle\left\langle\Gamma_{i}^{2}, C^{\text {aff }}\right\rangle\right\rangle} \quad \stackrel{\psi}{\rightarrow} \mathfrak{S}_{n} \rightarrow 1 .
$$

We will see in the proof of Theorem 3.6 that this sequence splits. Moreover, this short exact sequence is completely determined by $\pi_{1}\left(X^{\text {aff }}\right)$ and $n$. However, first we need

Lemma 3.5. The surjective homomorphism of Lemma 3.2 from $\psi^{-1}\left(\mathfrak{S}_{n-1}\right)$ onto $\pi_{1}\left(X^{\mathrm{aff}}\right)$ factors over the quotient by $C^{\text {aff }}$.

Proof. Without loss of generality we may assume that $\mathfrak{S}_{n-1}$ is the subgroup of $\mathfrak{S}_{n}$ fixing the first letter. We have to show that $C^{\text {aff }}$ is contained in $G_{n-1}$ : If one of the transpositions $\psi\left(\gamma \Gamma_{i} \gamma^{-1}\right)$ or $\psi\left(\Gamma_{j}\right)$ lies in $\mathfrak{S}_{n-1}$, this is true.

Hence we may assume that both transpositions do not lie in $\mathfrak{S}_{n-1}$. But this means that they have precisely the first letter in common. We write $a:=\gamma \Gamma_{i} \gamma^{-1}$ and $b:=\Gamma_{j}$. We have to show that $\langle a, b\rangle$ lies in $G_{n-1}$. Modulo $G_{n}$ the elements $a$ and $b$ equal their inverses, and so $\langle a, b\rangle$ is congruent to $\left(a b a^{-1}\right) \cdot\left(b^{-1} a^{-1} b\right)$. Both factors lie in $G_{n-1}$, and so the same is true for the product.

We now come to our first main result.

Theorem 3.6. For a good generic projection $f: X \rightarrow \mathbb{P}^{2}$ of degree $n$ there exists an isomorphism

$$
\pi_{1}\left(X_{\text {gal }}^{\mathrm{aff}}\right) / C^{\mathrm{aff}} \cong \widetilde{\mathcal{K}}\left(\pi_{1}\left(X^{\mathrm{aff}}\right), n\right)
$$

Proof. We consider the short exact sequence (5). Let us denote the group in the middle by $A$. It is generated by the $\Gamma_{i}$ 's, $i=1, \ldots, \operatorname{deg} D$. We have already seen that the $\Gamma_{i}$ 's and hence also their conjugates map to transpositions under $\psi$.

First, we split (5): For each transposition $\tau$ in $\mathfrak{S}_{n}$ we choose a conjugate $s(\tau)$ of some $\Gamma_{i}$ in $A$ that maps to this transposition under $\psi$, i.e., $\psi(s(\tau))=\tau$. Since we quotiented by $C^{\text {aff }}$, this map $s$, which is defined on transpositions, extends to a homomorphism

$$
s: \mathfrak{S}_{n} \rightarrow A \stackrel{\text { def }}{=} \pi_{1}\left(\mathbb{A}^{2}-D, x_{0}\right) /\left\langle\left\langle\Gamma_{i}^{2}, C^{\text {aff }}\right\rangle\right\rangle
$$

that splits $\psi$.

Next, we want to show that $A$ is a quotient of $\mathcal{S}_{n}(d)$ : We choose for every $\Gamma_{i}$ an element $\sigma_{i}$ in $\mathfrak{S}_{n}$ such that $\sigma_{i} \psi\left(\Gamma_{i}\right) \sigma_{i}{ }^{-1}=(12)$. We define

$$
s_{i}:=s\left(\sigma_{i}\right) \cdot \Gamma_{i} \cdot s\left(\sigma_{i}\right)^{-1}, \quad i=1, \ldots, \operatorname{deg} D .
$$


Clearly, $A$ is generated by the $s_{i}$ 's and $s\left(\mathfrak{S}_{n}\right)$. Recall Definition 2.10, where we defined the group $\mathcal{S}_{n}(d)$ in terms of $d$ generators $s_{i}$ and the group $\mathfrak{S}_{n}$ and relations between them. We define a map

$$
\begin{aligned}
& \mathcal{S}_{n}(d) \quad \rightarrow \quad A, \\
& s_{i} \quad \mapsto \quad s_{i}, \quad i=1, \ldots, d, \\
& \sigma \quad \mapsto s(\sigma) \quad \forall \sigma \in \mathfrak{S}_{n} .
\end{aligned}
$$

By definition of $C^{\text {aff }}$, this map extends to a surjective homomorphism of groups.

We let $F_{d}$ with $d=\operatorname{deg} D$ be the free group with basis $\Gamma_{i}, i=1, \ldots, d$. The group $\pi_{1}\left(\mathbb{A}^{2}-D, x_{0}\right)$ is a quotient of $F_{d}$ by a normal subgroup $R$; cf. Lemma 3.1. We factor this presentation via

$$
\begin{array}{cccccc}
F_{d} * \mathfrak{S}_{n} & \stackrel{\phi}{ } & \mathcal{S}_{n}(d) & \rightarrow & A, & \\
\Gamma_{i} & \mapsto & s\left(\sigma_{i}\right)^{-1} s_{i} s\left(\sigma_{i}\right) & \mapsto & \Gamma_{i}, & i=1, \ldots, d, \\
\sigma & \mapsto & \sigma & \mapsto & s(\sigma) & \forall \sigma \in \mathfrak{S}_{n} .
\end{array}
$$

By Theorem 2.11, the group $\mathcal{S}_{n}(d)$ is isomorphic to $\mathcal{E}\left(F_{d-1}, n\right)$, where $F_{d-1}$ is the free group of rank $d-1$. We will tacitly use this isomorphism. In particular, it makes sense to speak of affine subgroups of $\mathcal{S}_{n}(d)$; cf. Definition 2.8.

The group $A$ is a semidirect product of $K:=\pi_{1}\left(X^{\text {aff }}\right) / C^{\text {aff }}$ by $\mathfrak{S}_{n}$. Let $\varphi$ be the action of $\mathfrak{S}_{n}$ on the kernel $K$. For $k \in K$ and $\sigma \in \mathfrak{S}_{n}$, we write $[k, \sigma]$ for $k \cdot \varphi(\sigma)\left(k^{-1}\right)$. Let $G_{n-1}^{\prime}$ be the image of $G_{n-1}$ in $A$; cf. Lemma 3.2. It is easy to see that $\left[K, \mathfrak{S}_{n-1}\right]$ is equal to the intersection $G_{n-1}^{\prime} \cap K$. By Lemma 3.2 and Lemma 3.5. the quotient $\psi^{-1}\left(\mathfrak{S}_{n-1}\right) / G_{n-1}^{\prime}$ is isomorphic to $\pi_{1}\left(X^{\text {aff }}\right)$. Hence,

$$
K /\left[K, \mathfrak{S}_{n-1}\right] \cong \psi^{-1}\left(\mathfrak{S}_{n-1}\right) / G_{n-1}^{\prime} \cong \pi_{1}\left(X^{\mathrm{aff}}\right),
$$

where the first isomorphism follows from the second homomorphism theorem.

Consider the normal subgroup $R^{\prime}:=\phi(R)$ of $\mathcal{S}_{n}(d)$. We want to show that $R^{\prime}$ is an affine subgroup of $\mathcal{S}_{n}(d)$. By Lemma 3.1, the group $R$ is the normal closure of elements of a very special type. It is easy to check that these special elements map to elements of the form $\left(1, \ldots, r, 1, \ldots, r^{-1}, 1, \ldots\right)$ under $\phi$ and the isomorphism of Theorem 2.11. Clearly, such elements generate $R^{\prime}$. If we choose another embedding of $\mathfrak{S}_{n-1}$ into $\mathfrak{S}_{n}$, then formula (6) still holds true. This implies that $R^{\prime}$ is $\mathfrak{S}_{n^{-}}$ invariant. Hence, $R^{\prime}$ is an affine subgroup.

By Theorem 2.9, the group $K$ is isomorphic to $\widetilde{\mathcal{K}}(G, n)$ for a group $G$. By Corollary 2.5 this group $G$ is isomorphic to $K /\left[K, \mathfrak{S}_{n-1}\right]$. Applying (6), we obtain

$$
\pi_{1}\left(X_{\text {gal }}^{\mathrm{aff}}\right) / C^{\mathrm{aff}} \cong \widetilde{\mathcal{K}}\left(\pi_{1}\left(X^{\mathrm{aff}}\right), n\right) .
$$

Corollary 3.7. Let $\overline{C^{\text {aff }}}$ be the image of $C^{\text {aff }}$ in the Abelianisation of $\pi_{1}\left(X_{\text {gal }}^{\text {aff }}\right)$, i.e., in $H_{1}\left(X_{\text {gal }}^{\mathrm{aff}}, \mathbb{Z}\right)$. Then there exists a non-canonical isomorphism

$$
H_{1}\left(X_{\text {gal }}^{\text {aff }}, \mathbb{Z}\right) / \overline{C^{\text {aff }}} \cong H_{1}\left(X^{\text {aff }}, \mathbb{Z}\right)^{n-1} .
$$

Proof. This follows from Corollary 2.7 applied to Theorem 3.6 


\section{The fundamental Group of the Galois Closure}

We keep all notation introduced so far. We assume furthermore that the $\Gamma_{i}$ 's form a good ordered system of generators, i.e., that $\delta:=\Gamma_{1} \cdot \ldots \cdot \Gamma_{d}$ with $d=\operatorname{deg} D$ is homotopic to a loop around infinity in $\ell$.

Before stating our main results, we have to prove a couple of technical lemmas.

Lemma 4.1. The element $\delta$ lies in $\operatorname{ker} \psi$ and the centre of $\pi_{1}\left(\mathbb{A}^{2}-D, x_{0}\right)$. There are two short exact and central sequences

$$
\begin{aligned}
& 0 \rightarrow\langle\delta\rangle \rightarrow \pi_{1}\left(X_{\mathrm{gal}}^{\mathrm{aff}}\right) \rightarrow \pi_{1}\left(X_{\mathrm{gal}}\right) \rightarrow 1 \\
& 0 \rightarrow\langle\bar{\delta}\rangle \rightarrow \pi_{1}\left(X^{\mathrm{aff}}\right) \rightarrow \pi_{1}(X) \rightarrow 1
\end{aligned}
$$

Here, we denote the image of $\delta$ under $\operatorname{ker} \psi \rightarrow \pi_{1}\left(X_{\text {gal }}^{\text {aff }}\right)$ again by $\delta$, and its image under $\psi^{-1}\left(\mathfrak{S}_{n-1}\right) \rightarrow \pi_{1}\left(X^{\mathrm{aff}}\right)$ by $\bar{\delta}$.

Proof. By a theorem of Oka, $\delta$ is a central element; cf. [FL, Corollary 8.4]. Of course, $\delta$ remains central in every subquotient of $\pi_{1}\left(\mathbb{A}^{2}-D\right)$.

The subgroup generated by $\delta$ is normal and generates the kernel of the map from $\pi_{1}\left(\mathbb{A}^{2}-D\right)$ onto $\pi_{1}\left(\mathbb{P}^{2}-D\right)$. Chasing through the commutative diagram

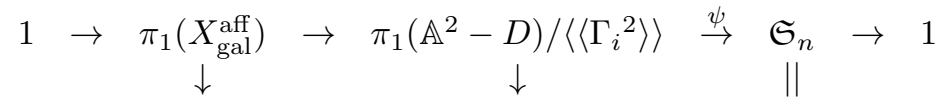

$$
\begin{aligned}
& 1 \rightarrow \pi_{1}\left(X_{\text {gal }}\right) \quad \rightarrow \quad \pi_{1}\left(\mathbb{P}^{2}-D\right) /\left\langle\left\langle\Gamma_{i}^{2}\right\rangle\right\rangle \stackrel{\psi}{\rightarrow} \mathfrak{S}_{n} \rightarrow 1
\end{aligned}
$$

we obtain the first exact sequence.

For the second statement we exhibit both $\pi_{1}\left(X^{\text {aff }}\right)$ and $\pi_{1}(X)$ as quotients of $\psi^{-1}\left(\mathfrak{S}_{n-1}\right)$ by $G_{n-1}$; cf. Lemma 3.2 . Then we proceed as before.

Lemma 4.2. We consider $\mathcal{K}\left(\pi_{1}\left(X^{\mathrm{aff}}\right), n\right)$ as a subgroup of $\pi_{1}\left(X^{\mathrm{aff}}\right)^{n}$. Under the map (2) the element $\delta$ maps

$$
\begin{array}{ccccc}
\pi_{1}\left(X_{\text {gal }}^{\mathrm{aff}}\right) / C^{\mathrm{aff}} & \cong & \widetilde{\mathcal{K}}\left(\pi_{1}\left(X^{\mathrm{aff}}\right), n\right) & \rightarrow & \mathcal{K}\left(\pi_{1}\left(X^{\mathrm{aff}}\right), n\right), \\
\delta & \mapsto & \delta^{\prime} & \mapsto & (\bar{\delta}, \ldots, \bar{\delta})
\end{array}
$$

where $\delta$ and $\bar{\delta}$ are as in Lemma 4.1.

In particular, $\bar{\delta}^{n}$ is an element of the kernel $\operatorname{ker}\left(\pi_{1}\left(X^{\mathrm{aff}}\right) \rightarrow \pi_{1}\left(X^{\mathrm{aff}}\right)^{\mathrm{ab}}\right)$.

Proof. The group $E:=\widetilde{\mathcal{K}}\left(\pi_{1}\left(X^{\text {aff }}\right), n\right) \rtimes \mathfrak{S}_{n}$ is a quotient of $\pi_{1}\left(\mathbb{A}^{2}-D\right)$ by Theorem 3.6. As $\delta$ is central in $\pi_{1}\left(\mathbb{A}^{2}-D\right)$, its image $\delta^{\prime}$ is a central in $E$. In particular, $\delta^{\prime}$ is $\mathfrak{S}_{n}$-invariant. The map from $\widetilde{\mathcal{K}}\left(\pi_{1}\left(X^{\text {aff }}\right), n\right)$ onto $\mathcal{K}\left(\pi_{1}\left(X^{\text {aff }}\right), n\right)$ is $\mathfrak{S}_{n}$-equivariant. Thus, $\delta^{\prime}$ maps to a $\mathfrak{S}_{n}$-invariant element, i.e., to an element of the diagonal in $\pi_{1}\left(X^{\text {aff }}\right)^{n}$.

There are two maps from $\pi_{1}\left(X_{\text {gal }}^{\text {aff }}\right)$ onto $\pi_{1}\left(X^{\text {aff }}\right)$ : One is the map from Lemma 4.2 composed with the projection onto the first factor from $\pi_{1}\left(X^{\text {aff }}\right)^{n}$ onto $\pi_{1}\left(X^{\text {aff }}\right)$. We have to show that $\delta$ maps to $\bar{\delta}$ under this map. The second one is the map induced from Lemma 3.2. Under this second map, the element $\delta$ maps to $\bar{\delta}$. Going through the construction of the first map in the proof of Theorem $\underline{3.6}$, we see that it is constructed via Corollary 2.5, which is compatible with Lemma 3.2. Hence, the first and the second map coincide. In particular, $\delta$ maps to $\bar{\delta}$. 
The element $(\bar{\delta}, \ldots, \bar{\delta})$ lies in $\mathcal{K}\left(\pi_{1}\left(X^{\text {aff }}\right), n\right)$. By definition, $\bar{\delta}^{n}$ lies in

$$
\operatorname{ker}\left(\pi_{1}\left(X^{\mathrm{aff}}\right) \rightarrow \pi_{1}\left(X^{\mathrm{aff}}\right)^{\mathrm{ab}}\right) .
$$

Lemma 4.3. Let $X$ be a smooth projective surface and let $D$ be a smooth and ample divisor on it. If $\pi_{1}(X)$ is finite, then so is $\pi_{1}(X-D)$.

Moreover, if $X$ is simply connected, then $\pi_{1}(X-D)$ is a finite cyclic group whose order is equal to the divisibility index of $\mathcal{O}_{X}(D)$ in $\operatorname{Pic}(X)$.

Proof. Let us first assume that $\pi_{1}(X)$ is trivial. As an ample divisor, $D$ is connected. Thus, $D$ is irreducible, being connected and smooth. It follows that every finite cover of $X$ branched along $D$ is a cyclic Galois cover.

A cyclic cover branched along $D$ is given by a line bundle $\mathcal{F}$ together with an isomorphism $\mathcal{F}^{\otimes c} \cong \mathcal{O}_{X}(D)$. Hence the maximal finite quotient of $\pi_{1}(X-D)$ is cyclic of order equal to the divisibility index $d$ of $\mathcal{O}_{X}(D)$ in $\operatorname{Pic}(X)$.

By Nori's theorem [No, Corollary 2.5], the group $\pi_{1}(X-D)$ is finitely generated and Abelian. Hence this group is cyclic of order $d$. This proves the second assertion.

If $\pi_{1}(X)$ is finite, then the universal cover $\tilde{X}$ of $X$ is again a smooth projective surface. The inverse image $\tilde{D}$ of $D$ on $\tilde{X}$ is a smooth and ample divisor. Hence $\pi_{1}(\tilde{X}-\tilde{D})$ is a finite group by what we have just proved. But this latter group is a group of finite index in $\pi_{1}(X-D)$. Hence $\pi_{1}(X-D)$ is finite, which proves the first assertion.

The inclusion map of topological spaces induces a surjective homomorphism from $\pi_{1}\left(X_{\text {gal }}^{\text {aff }}\right)$ onto $\pi_{1}\left(X_{\text {gal }}\right)$. Recall that we defined (Definition 3.3) a certain subgroup $C^{\text {aff }}$ of $\pi_{1}\left(X_{\text {gal }}^{\text {aff }}\right)$ and determined the structure of $\pi_{1}\left(X_{\text {gal }}^{\text {aff }}\right) / C^{\text {aff }}$.

Definition 4.4. We denote the image of $C^{\text {aff }}$ in $\pi_{1}\left(X_{\text {gal }}\right)$ by $C^{\text {proj }}$.

Clearly, if $C^{\text {aff }}$ is trivial, then the same is true for $C^{\text {proj }}$. As already noted above, $C^{\text {aff }}$ is trivial in all known examples.

Before giving the structure of $\pi_{1}\left(X_{\text {gal }}\right) / C^{\text {proj }}$, we have to introduce a little bit of notation. First we define

$$
K:=\operatorname{ker}\left(\pi_{1}\left(X^{\text {aff }}\right) \rightarrow \pi_{1}(X)\right),
$$

which is a cyclic group. For a natural number $m \geq 1$ we define the homomorphism

$$
\begin{aligned}
\kappa_{m}: K^{m} & \rightarrow \pi_{1}\left(X^{\mathrm{aff}}\right)^{\mathrm{ab}} \\
\left(k_{1}, \ldots, k_{m}\right) & \mapsto \sum_{i=1}^{m} \bar{k}_{i}
\end{aligned}
$$

We are now ready to state our main result on $\pi_{1}\left(X_{\text {gal }}\right) / C^{\text {proj }}$.

Theorem 4.5. There exists a cyclic group $Z$ and a central short exact sequence

$$
0 \rightarrow \frac{H_{2}\left(\pi_{1}\left(X^{\mathrm{aff}}\right), \mathbb{Z}\right)}{Z} \rightarrow \pi_{1}\left(X_{\text {gal }}\right) / C^{\text {proj }} \rightarrow G \rightarrow 1,
$$

where $G$ is a group that is itself a central extension

$$
0 \rightarrow \operatorname{ker} \kappa_{n-1} \rightarrow G \rightarrow \mathcal{K}\left(\pi_{1}(X), n\right) \rightarrow 1 .
$$

The kernel in (7) lies inside the commutator subgroup of $\mathcal{K}\left(\pi_{1}(X), n\right)$. 
Proof. By Theorem 3.6 the group $\pi_{1}\left(X_{\text {gal }}^{\text {aff }}\right) / C^{\text {aff }}$ is isomorphic to $\widetilde{\mathcal{K}}\left(\pi_{1}\left(X^{\text {aff }}\right), n\right)$. By Theorem 2.4 this group is a central extension

$$
0 \rightarrow H_{2}\left(\pi_{1}\left(X^{\mathrm{aff}}\right), \mathbb{Z}\right) \rightarrow \pi_{1}\left(X_{\text {gal }}^{\mathrm{aff}}\right) / C^{\mathrm{aff}} \stackrel{\psi}{\rightarrow} \mathcal{K}\left(\pi_{1}\left(X^{\mathrm{aff}}\right), n\right) \rightarrow 1,
$$

where the kernel lies inside the commutator subgroup.

To obtain $\pi_{1}\left(X_{\text {gal }}\right) / C^{\text {proj }}$, we take the quotient of $\pi_{1}\left(X_{\text {gal }}^{\text {aff }}\right) / C^{\text {aff }}$ by $\langle\delta\rangle$. We let $Z$ be the intersection of $\langle\delta\rangle$ with $H_{2}\left(\pi_{1}\left(X^{\text {aff }}\right), \mathbb{Z}\right)$. Thus, $\pi_{1}\left(X_{\text {gal }}\right) / C^{\text {proj }}$ is a central extension of $H_{2}\left(\pi_{1}\left(X^{\text {aff }}\right), \mathbb{Z}\right) / Z$ by $G:=\mathcal{K}\left(\pi_{1}\left(X^{\text {aff }}\right), n\right) / \psi(\delta)$. This already gives the first exact sequence.

By definition, $K$ is the kernel $\operatorname{ker}\left(\pi_{1}\left(X^{\text {aff }}\right) \rightarrow \pi_{1}(X)\right)$. Using the definition of $\mathcal{K}(-, n)$ it is not hard to see that the kernel of $\mathcal{K}\left(\pi_{1}\left(X^{\text {aff }}\right), n\right) \rightarrow \mathcal{K}\left(\pi_{1}(X), n\right)$ is equal to ker $\kappa_{n}$. The image of $\delta$ in $\pi_{1}(X)$ is trivial, and so $\psi(\delta)$ lies in ker $\kappa_{n}$. Moreover, by Lemma 4.2. the image $\psi(\delta)$ generates the subgroup of ker $\kappa_{n}$ that is equal to $\Delta(K)$, where $\Delta: K \rightarrow K^{n}$ is the map that sends $k$ to $(k, \ldots, k)$. It is not difficult to see that ker $\kappa_{n} / \Delta(K)$ is isomorphic to ker $\kappa_{n-1}$.

Thus $G$ is an extension of ker $\kappa_{n} / \psi(\delta) \cong \operatorname{ker} \kappa_{n-1}$ by $\mathcal{K}\left(\pi_{1}(X), n\right)$.

Corollary 4.6. If $\pi_{1}(X)$ is finite, then so is $\pi_{1}\left(X_{\text {gal }}\right) / C^{\text {proj }}$.

Proof. If $\pi_{1}(X)$ is finite, then so is $\pi_{1}\left(X^{\text {aff }}\right)$ by Lemma 4.3. Thus, $\pi_{1}\left(X^{\text {aff }}\right)^{n}$ and $H_{2}\left(\pi_{1}\left(X^{\text {aff }}\right), \mathbb{Z}\right)$ are finite. In particular, $\widetilde{\mathcal{K}}\left(\pi_{1}\left(X^{\text {aff }}\right), n\right)$ is finite by Theorem 2.4.

Hence $\pi_{1}\left(X_{\text {gal }}^{\text {aff }}\right) / C^{\text {aff }}$ is finite by Theorem 3.6. Since $\pi_{1}\left(X_{\text {gal }}\right) / C^{\text {proj is a quotient }}$ of this latter group, it is also finite.

Corollary 4.7. If $\pi_{1}(X)$ is nilpotent of class $c$, then $\pi_{1}\left(X_{\text {gal }}\right) / C^{\text {proj }}$ is nilpotent of class at least $c$ and at most $c+2$.

Proof. If $H:=\pi_{1}\left(X_{\text {gal }}\right)$ is nilpotent of class $c$, then the same is true for $H^{n}$. Since $\mathcal{K}(H, n)$ is a subgroup of $H^{n}, \mathcal{K}(H, n)$ is also nilpotent of class at most $c$. By Theorem 4.5, we obtain $G:=\pi_{1}\left(X_{\text {gal }}\right) / C^{\text {proj }}$ as two successive central extensions of $\mathcal{K}(H, n)$ by Abelian groups. Hence $K$ is nilpotent of class at most $c+2$.

There is a surjective map from $G$ onto $\mathcal{K}(H, n)$, which has a surjective map onto $H$. In particular, $G$ is of class at least $c$.

Proposition 4.8. Let $\overline{C^{\text {proj }}}$ be the image of $C^{\text {proj }}$ in the Abelianisation of $\pi_{1}\left(X_{\text {gal }}\right)$, i.e., in $H_{1}\left(X_{\text {gal }}, \mathbb{Z}\right)$. Then there exists a non-canonical isomorphism

$$
H_{1}\left(X_{\text {gal }}, \mathbb{Z}\right) / \overline{C^{\text {proj }}} \cong H_{1}(X, \mathbb{Z}) \oplus\left(H_{1}\left(X^{\text {aff }}, \mathbb{Z}\right)\right)^{n-2} .
$$

The group $H_{1}\left(X^{\mathrm{aff}}, \mathbb{Z}\right)$ is an extension of a finite cyclic group (of order dividing $n$ ) by $H_{1}(X, \mathbb{Z})$.

Proof. By Lemma 4.1 $\delta$ generates the $\operatorname{kernel} \operatorname{ker}\left(\pi_{1}\left(X_{\text {gal }}^{\text {aff }}\right) \rightarrow \pi_{1}\left(X_{\text {gal }}\right)\right)$. It follows that the image of $\delta$ in $H_{1}\left(X_{\mathrm{gal}}^{\text {aff }}\right)$ generates the kernel of the induced surjective homomorphism from $H_{1}\left(X_{\text {gal }}^{\text {aff }}\right)$ onto $H_{1}\left(X_{\text {gal }}\right)$. Similarly, the image of $\bar{\delta}$ in $H_{1}\left(X^{\text {aff }}\right)$ generates the kernel from $H_{1}\left(X^{\text {aff }}\right)$ onto $H_{1}(X)$. By abuse of notation we will write $\delta$ and $\bar{\delta}$ also for their images in the Abelianised groups. By Lemma 4.2, the order of $\bar{\delta}$ (as an element of $H_{1}\left(X^{\text {aff }}\right)$ ) divides $n$.

By Corollary 3.7 there exists an embedding

$$
\imath: H_{1}\left(X_{\text {gal }}^{\text {aff }}\right) / \overline{C^{\text {aff }}} \rightarrow\left(H_{1}\left(X^{\text {aff }}\right)\right)^{n}
$$


with image $\mathcal{K}\left(H_{1}\left(X^{\text {aff }}\right), n\right)$. We denote by $p$ the projection of $H_{1}\left(X^{\text {aff }}\right)^{n}$ onto its last $n-1$ factors. Then $p$ induces an isomorphism of $\mathcal{K}\left(H_{1}\left(X^{\text {aff }}\right), n\right)$ with $H_{1}\left(X^{\text {aff }}\right)^{n-1}$; cf. Proposition 2.2. By Lemma 4.2, the element $\delta$ maps to $(\bar{\delta}, \ldots, \bar{\delta})$ under $\imath$. Thus, $p \circ \imath(\delta)$ is equal to $(\bar{\delta}, \ldots, \bar{\delta})$ in $\pi_{1}\left(X^{\text {aff }}\right)^{n-1}$.

To obtain $H_{1}\left(X_{\text {gal }}\right) / \overline{C^{\text {proj }}}$, we have to form the quotient of $H_{1}\left(X^{\text {aff }}\right)^{n-1}$ by $\langle\delta\rangle$. The quotient of $H_{1}\left(X^{\text {aff }}\right)$ by $\bar{\delta}$ is isomorphic to $H_{1}(X)$. The image of $\delta$ in $H_{1}\left(X^{\text {aff }}\right)^{n-1}$ lies on the diagonal $(\bar{\delta}, \ldots, \bar{\delta})$.

If $G$ is an Abelian group, $\Delta: G \rightarrow G^{n-1}$ is the map that sends $g$ to $(g, \ldots, g)$ and if $N$ is a subgroup of $G$, then $G^{n-1} / \Delta(N)$ is isomorphic to $G^{n-2} \oplus G / N$. The proposition then follows if we apply this fact to $G:=H_{1}\left(X^{\text {aff }}\right)$ and $N:=\langle\delta\rangle$.

Corollary 4.9. The rank of $H_{1}\left(X_{\text {gal }}, \mathbb{Z}\right) / \overline{C^{\text {proj }}}$ as an Abelian group is equal to $(n-1)$ times the rank of $H_{1}(X, \mathbb{Z})$.

Corollary 4.10. Let $X$ be a surface such that $H_{1}(X, \mathbb{Z}) \cong \mathbb{Z}^{b_{1}}$. Let $\mathcal{L}$ be a sufficiently ample line bundle on $X$ and let $f: X \rightarrow \mathbb{P}^{2}$ be a good generic projection with respect to $\mathcal{L}$. We denote by $n$ the self-intersection number and by $d$ the divisibility index of $\mathcal{L}$ in $\operatorname{Pic}(X)$. Then there are isomorphisms

$$
\begin{aligned}
& H_{1}\left(X_{\text {gal }}^{\text {aff }}, \mathbb{Z}\right) / \overline{C^{\text {aff }}} \cong(\mathbb{Z} / d \mathbb{Z})^{n-1} \oplus \mathbb{Z}^{b_{1}(n-1)}, \\
& H_{1}\left(X_{\text {gal }}, \mathbb{Z}\right) / \overline{C^{\text {proj }}} \cong(\mathbb{Z} / d \mathbb{Z})^{n-2} \oplus \mathbb{Z}^{b_{1}(n-1)} .
\end{aligned}
$$

These isomorphisms are not canonical.

Proof. Abelianising the short exact sequence of Lemma 4.1 we obtain

$$
0 \rightarrow \phi(Z) \rightarrow \pi_{1}\left(X^{\mathrm{aff}}\right)^{\mathrm{ab}} \rightarrow \pi_{1}(X)^{\mathrm{ab}} \rightarrow 0,
$$

where $Z:=\langle\bar{\delta}\rangle$ with $\bar{\delta}$ the loop around infinity as in Lemma 4.1 and where $\phi$ denotes the map from $\pi_{1}\left(X^{\text {aff }}\right)$ onto its Abelianisation. As $\pi_{1}(X)^{\text {ab }} \cong \mathbb{Z}^{b_{1}}$ is a free Abelian group, this sequence splits, i.e., $\pi_{1}\left(X^{\mathrm{aff}}\right)^{\mathrm{ab}} \cong \phi(Z) \oplus \mathbb{Z}^{b_{1}}$.

Hence $\phi(Z)$ is a quotient of $\pi_{1}\left(X^{\text {aff }}\right)$. As in the proof of Lemma 4.3 we conclude that the order of $\phi(Z)$ is equal to the divisibility index $d$ of $\mathcal{L}$.

Using Corollary 3.7 and Proposition 4.8 completes the proof.

Proposition 4.11. Let $X$ be a simply connected surface and let $\mathcal{L}$ be a line bundle that defines a good generic projection. We let $d$ be the divisibility index of $\mathcal{L}$ in $\operatorname{Pic}(X)$. We denote the self-intersection of $\mathcal{L}$ by $n$. Then there exist isomorphisms

$$
\begin{aligned}
& \pi_{1}\left(X_{\text {gal }}^{\text {aff }}\right) / C^{\text {aff }} \cong(\mathbb{Z} / d \mathbb{Z})^{n-1}, \\
& \pi_{1}\left(X_{\text {gal }}\right) / C^{\text {proj }} \cong(\mathbb{Z} / d \mathbb{Z})^{n-2} .
\end{aligned}
$$

These isomorphisms are not canonical.

Proof. Let $f: X \rightarrow \mathbb{P}^{2}$ be a good generic projection corresponding to $\mathcal{L}$. Clearly, the degree $n=\operatorname{deg} f$ is equal to the self-intersection of $\mathcal{L}$.

The inverse image $H:=f^{-1}(\ell)$ of the line at infinity is a smooth and ample divisor on $X$ by Bertini's theorem. By definition of $f$, the line bundle $\mathcal{O}_{X}(H)$ is isomorphic to $\mathcal{L}$. Since $X^{\text {aff }}=X-H$, Lemma 4.3 tells us that $\pi_{1}\left(X^{\text {aff }}\right)$ is cyclic of order $d$.

Applying Theorem 3.6 and Corollary 2.6, we obtain

$$
\pi_{1}\left(X_{\text {gal }}^{\text {aff }}\right) / C^{\text {aff }} \cong \widetilde{\mathcal{K}}\left(\pi_{1}\left(X^{\text {aff }}\right), n\right) \cong(\mathbb{Z} / d \mathbb{Z})^{n-1} .
$$


In particular, $\pi_{1}\left(X_{\text {gal }}^{\text {aff }}\right) / C^{\text {aff }}$ is Abelian. So, the same is true for $\pi_{1}\left(X_{\text {gal }}\right) / C^{\text {proj }}$. Thus we can apply Proposition 4.8 to determine $\pi_{1}\left(X_{\text {gal }}\right) / C^{\text {proj }}$.

If $X$ is not simply connected, we now determine $H_{2}\left(\pi_{1}\left(X^{\text {aff }}\right), \mathbb{Z}\right)$ up to extension. This will be useful for the examples in the following section.

Lemma 4.12. There exists a short exact sequence of Abelian groups

$$
\begin{aligned}
0 \rightarrow \frac{\pi_{1}(X)^{\mathrm{ab}} \otimes_{\mathbb{Z}} K}{i\left(H_{3}\left(\pi_{1}(X), \mathbb{Z}\right)\right)} & \rightarrow \quad H_{2}\left(\pi_{1}\left(X^{\mathrm{aff}}\right), \mathbb{Z}\right) \\
& \rightarrow \operatorname{ker}\left(H_{2}\left(\pi_{1}(X), \mathbb{Z}\right) \rightarrow K\right) \rightarrow 0 .
\end{aligned}
$$

Proof. The kernel $K$ is cyclic. Hence $H_{i}(K, \mathbb{Z})=0$ for $i \neq 0,1$. This implies that the spectral sequence

$$
E_{p q}^{2}:=H_{p}\left(\pi_{1}(X), H_{q}(K, \mathbb{Z})\right) \Rightarrow H_{p+q}\left(\pi_{1}\left(X^{\mathrm{aff}}\right), \mathbb{Z}\right)
$$

has only two rows. This yields a long exact sequence

$$
\begin{aligned}
& \ldots \rightarrow H_{3}\left(\pi_{1}(X), \mathbb{Z}\right) \stackrel{\iota}{\rightarrow} H_{1}\left(\pi_{1}(X), K\right) \rightarrow H_{2}\left(\pi_{1}\left(X^{\text {aff }}\right), \mathbb{Z}\right) \rightarrow H_{2}\left(\pi_{1}(X), \mathbb{Z}\right) \\
& \rightarrow H_{1}(K, \mathbb{Z}) \rightarrow H_{1}\left(\pi_{1}\left(X^{\text {aff }}\right), \mathbb{Z}\right) \rightarrow \ldots
\end{aligned}
$$

Using the universal coefficient formula we obtain isomorphisms

$$
H_{1}\left(\pi_{1}(X), K\right) \cong H_{1}\left(\pi_{1}(X), \mathbb{Z}\right) \otimes_{\mathbb{Z}} K \cong \pi_{1}(X)^{\mathrm{ab}} \otimes_{\mathbb{Z}} K .
$$

This yields the short exact sequence (8).

We end this section by a remark on Teicher's conjecture. In [Te, Teicher observed that $\pi_{1}\left(\mathbb{A}^{2}-D\right)$ is virtually solvable in some examples and asked whether this might be a general phenomenon.

Proposition 4.13. If $\pi_{1}(X)$ is not virtually solvable, then neither is $\pi_{1}\left(\mathbb{A}^{2}-D\right)$.

Proof. This follows from the fact that $\pi_{1}\left(\mathbb{A}^{2}-D\right)$ has $\widetilde{\mathcal{K}}\left(\pi_{1}\left(X^{\text {aff }}, n\right)\right)$ as a subquotient. This latter group has $\mathcal{K}\left(\pi_{1}(X), n\right)$ as a quotient which has $\pi_{1}(X)$ as a quotient. Hence, if $\pi_{1}\left(\mathbb{A}^{2}-D\right)$ were virtually solvable, then $\pi_{1}(X)$ would have to be as well.

The fundamental group of a ruled surface over a curve of genus $\geq 2$ contains a free group of rank 2 . Hence $\pi_{1}\left(\mathbb{A}^{2}-D\right)$ for the generic projection from a ruled surface over a curve of genus $\geq 2$ is not virtually solvable.

\section{EXAMPLES}

In this section we discuss all the examples that have been computed so far. We will see that Question 3.4 has a positive answer in all cases. We also show how methods from homological algebra simplify and clarify the computations.

Minimal rational surfaces. The fundamental groups of Galois closures of generic projections from $\mathbb{P}^{2}, \mathbb{P}^{1} \times \mathbb{P}^{1}$ and the Hirzebruch surfaces $\mathbb{F}_{e}$ have been calculated in a series of papers by Moishezon, Teicher and Robb. 
Proposition 5.1. Let $\mathcal{L}:=\mathcal{O}_{\mathbb{P}^{2}}(k)$ with $k \geq 5$ on $X:=\mathbb{P}^{2}$. Let $f: X \rightarrow \mathbb{P}^{2}$ be a good generic projection with respect to a generic 3 -dimensional subspace of $H^{0}(X, \mathcal{L})$ and let $X_{\mathrm{gal}}$ be the corresponding Galois closure. Then there are isomorphisms

$$
\begin{aligned}
& \pi_{1}\left(X_{\text {gal }}^{\text {aff }}\right) / C^{\text {aff }} \cong(\mathbb{Z} / k \mathbb{Z})^{k^{2}-1}, \\
& \pi_{1}\left(X_{\text {gal }}\right) / C^{\text {proj }} \cong(\mathbb{Z} / k \mathbb{Z})^{k^{2}-2} .
\end{aligned}
$$

Proof. For $k \geq 5$ the line bundle $\mathcal{L}$ is sufficiently ample by Remark 1.2, The morphism $f: X \rightarrow \mathbb{P}^{2}$ has degree equal to the self-intersection of $\mathcal{L}$, which is $k^{2}$. The divisibility index of $\mathcal{L}$ in $\operatorname{Pic}(X)$ is equal to $k$. Then we apply Proposition 4.11.

Remark 5.2. The computations in MoTe2] show that the two groups $C^{\text {aff }}$ and $C^{\text {proj }}$ are trivial already for $k \geq 3$, i.e., Question 3.4 has a positive answer in these cases.

Remark 5.3. For $k=2$, the surface $X_{\text {gal }}$ is the Galois closure of a generic projection from the Veronese surface $V_{4}$ of degree 4 in $\mathbb{P}^{5}$. Moishezon and Teicher proved that $X_{\text {gal }}$ is an Abelian surface; cf. [MoTe2, Proposition 1]. In particular, $\pi_{1}\left(X_{\text {gal }}\right)$ is isomorphic to $\mathbb{Z}^{4}$, whereas we would only predict $(\mathbb{Z} / 2 \mathbb{Z})^{2}$ in this case (our results are not valid for projections of such small degree). However, this example should not be discouraging in view of Question 3.4. The surface $V_{4}$ has to be excluded from many statements in classical algebraic geometry. For example, a generic projection from $V_{4}$ is the only counterexample to Chisini's conjecture $[\mathrm{Ku}]$.

Proposition 5.4. Let $\mathcal{L}:=\mathcal{O}_{\mathbb{P}^{1} \times \mathbb{P}^{1}}(a, b)$ with $a, b \geq 5$ on $X:=\mathbb{P}^{1} \times \mathbb{P}^{1}$. Let $f: X \rightarrow \mathbb{P}^{2}$ be a good generic projection with respect to a generic 3 -dimensional subspace of $H^{0}(X, \mathcal{L})$ and let $X_{\text {gal }}$ be the corresponding Galois closure. Then there are isomorphisms

$$
\begin{aligned}
& \pi_{1}\left(X_{\text {gal }}^{\text {aff }}\right) / C^{\text {aff }} \cong(\mathbb{Z} / \operatorname{gcd}(a, b) \mathbb{Z})^{2 a b-1} \\
& \pi_{1}\left(X_{\text {gal }}\right) / C^{\text {proj }} \cong(\mathbb{Z} / \operatorname{gcd}(a, b) \mathbb{Z})^{2 a b-2}
\end{aligned}
$$

Proof. For $a, b \geq 5$ the line bundle $\mathcal{L}$ is sufficiently ample by Remark 1.2, The morphism $f$ has degree equal to the self-intersection of $\mathcal{L}$, which is $2 a b$. The divisibility index of $\mathcal{L}$ in $\operatorname{Pic}(X)$ is equal to $\operatorname{gcd}(a, b)$. Then we apply Proposition 4.11.

Remark 5.5. The results of [MoTe1] and [MoTe3] show that $C^{\text {aff }}$ and $C^{\text {proj }}$ are trivial, i.e., Question 3.4 has a positive answer in these cases.

Let us introduce some notation before computing the next example. We let $X:=\mathbb{F}_{e}:=\mathbb{P}\left(\mathcal{O}_{\mathbb{P}^{1}} \oplus \mathcal{O}_{\mathbb{P}^{1}}(-e)\right)$ be the $e$-th Hirzebruch surface. We regard $\mathbb{F}_{e}$ as a projectivised $\mathbb{P}^{1}$-bundle over $\mathbb{P}^{1}$. We denote by $F$ the class of a fibre and by $H$ the class of the tautological bundle inside $\operatorname{Pic}\left(\mathbb{F}_{e}\right)$. For $a \gg 0$ and $b \gg 0$ the bundle $\mathcal{L}:=\mathcal{O}_{\mathbb{F}_{e}}(a H+b F)$ will give rise to good generic projections.

Proposition 5.6. Let $\mathcal{L}:=\mathcal{O}_{\mathbb{F}_{e}}(a H+b F)$ be a sufficiently ample line bundle on $X:=\mathbb{F}_{e}$. Let $f: X \rightarrow \mathbb{P}^{2}$ be a good generic projection with respect to a generic 3 dimensional subspace of $H^{0}(X, \mathcal{L})$ and let $X_{\text {gal }}$ be the corresponding Galois closure. Then there are isomorphisms

$$
\begin{aligned}
& \pi_{1}\left(X_{\text {gal }}^{\text {aff }}\right) / C^{\text {aff }} \cong(\mathbb{Z} / \operatorname{gcd}(a, b) \mathbb{Z})^{2 a b+e a^{2}-1}, \\
& \pi_{1}\left(X_{\text {gal }}\right) / C^{\text {proj }} \cong(\mathbb{Z} / \operatorname{gcd}(a, b) \mathbb{Z})^{2 a b+e a^{2}-2} .
\end{aligned}
$$


Proof. The degree of $f$ is equal to the self-intersection of $\mathcal{L}$, which is $2 a b+e a^{2}$. The divisibility index of $\mathcal{L}$ in $\operatorname{Pic}(X)$ is equal to $\operatorname{gcd}(a, b)$. Then we apply Proposition 4.11 .

Remark 5.7. The results of $\mathrm{MTR}$ show that $C^{\text {aff }}$ and $C^{\text {proj }}$ are trivial, i.e., Question 3.4 has a positive answer in these cases.

Product of a curve with $\mathbb{P}^{1}$. The fundamental groups of the Galois closures of certain generic projections from $E \times \mathbb{P}^{1}$, where $E$ is an elliptic curve, have been computed by Amram, Goldberg, Teicher and Vishne. These groups are particularly interesting for us since this is the first time homological contributions enter the picture.

We let $C$ be a curve of genus $g \geq 1$ and $X:=C \times \mathbb{P}^{1}$. We let $\mathcal{F}$ be a very ample line bundle of degree $k$ on $C$. The line bundle $\mathcal{L}:=\mathcal{F} \otimes \mathcal{O}_{\mathbb{P}^{1}}(d)$ on $X$ is very ample. If we choose $k$ and $d$ sufficiently large, $\mathcal{L}$ gives rise to good generic projections $f: X \rightarrow \mathbb{P}^{2}$. The degree $n=\operatorname{deg} f$ is equal to the self-intersection of $\mathcal{L}$, which is equal to $2 d k$.

To apply our results, we have to compute $\pi_{1}\left(X^{\text {aff }}\right)$ and $H_{2}\left(\pi_{1}\left(X^{\text {aff }}\right), \mathbb{Z}\right)$.

Lemma 5.8. The group $\pi_{1}\left(X^{\text {aff }}\right)$ is an extension of $\mathbb{Z} / d \mathbb{Z}$ by $\pi_{1}(X) \cong \pi_{1}(C)$. If $d$ divides $k$, then this extension splits.

Moreover, there is an isomorphism $\pi_{1}\left(X^{\mathrm{aff}}\right)^{\mathrm{ab}} \cong(\mathbb{Z} / \operatorname{gcd}(k, d) \mathbb{Z}) \oplus \mathbb{Z}^{2 g}$.

Proof. We only prove the case $g=1$. For $g \geq 2$ we only have to replace $\mathbb{C}^{2}$ by the upper half-plane $\mathbb{H}$.

We let $H$ be a smooth section of $\mathcal{L}$. Then there is a short exact sequence

$$
0 \rightarrow K \rightarrow \pi_{1}\left(X^{\text {aff }}\right) \rightarrow \pi_{1}(X) \rightarrow 1,
$$

where $K$ is a cyclic group in the centre of $\pi_{1}\left(X^{\text {aff }}\right)$; cf. Lemma 4.1.

We let $\tilde{X}$ be the universal cover of $X$, i.e., $\tilde{X}$ is isomorphic to $\mathbb{C}^{2} \times \mathbb{P}^{1}$. The pull-back of $\mathcal{L}$ to $\tilde{X}$ is isomorphic to the line bundle $\tilde{\mathcal{L}}:=\mathcal{O}_{\mathbb{P}^{1}}(d) \otimes \mathcal{O}_{\mathbb{C}^{2}}$, as every line bundle on $\mathbb{C}^{2}$ is trivial.

The group $C$ corresponds to covers of $\tilde{X}$ ramified along the inverse image of $H$ on $\tilde{X}$. Arguing as in the proof of Lemma 4.3 we see that the order of $K$ is equal to the divisibility index of $\tilde{\mathcal{L}}$ on $\tilde{X}$, which is equal to $d$. Hence $K$ is cyclic of order $d$.

If $k$ is divisible by $d$, we can define a $d$-fold cover of $X$ by choosing a line bundle $\mathcal{F}$ such that $\mathcal{F}^{\otimes d} \cong \mathcal{L}$. Such a cover corresponds to a surjective homomorphism from $\pi_{1}(X-D)$ onto $K$ compatible with (9). So in this case the map from $K$ to $\pi_{1}\left(X^{\text {aff }}\right)$ is a split injection.

Abelianising (9) we obtain $\pi_{1}\left(X^{\text {aff }}\right)^{\text {ab }}$ as an extension of a finite cyclic group $K^{\prime}$ by $\mathbb{Z}^{2 g}$. This extensions splits since the quotient is free Abelian. Hence $K^{\prime}$ is a quotient of $\pi_{1}\left(X^{\mathrm{aff}}\right)^{\mathrm{ab}}$. The order of $K^{\prime}$ is given by the divisibility index of $\mathcal{L}$, which is equal to $\operatorname{gcd}(k, d)$.

Lemma 5.9. There is an isomorphism $H_{2}\left(\pi_{1}\left(X^{\text {aff }}\right), \mathbb{Z}\right) \cong(\mathbb{Z} / d \mathbb{Z})^{2 g} \oplus \mathbb{Z}$.

Proof. The curve $C$ has genus $g \geq 1$ and so it is an Eilenberg-MacLane space. Hence the group homology $H_{i}\left(\pi_{1}(C), \mathbb{Z}\right)$ is isomorphic to the singular homology $H_{i}(C, \mathbb{Z})$. In particular, the group $H_{3}\left(\pi_{1}(X), \mathbb{Z}\right)$ vanishes and $H_{2}\left(\pi_{1}(X), \mathbb{Z}\right) \cong \mathbb{Z}$. Moreover, $H_{1}\left(\pi_{1}(X), \mathbb{Z}\right) \cong \pi_{1}(X)^{\mathrm{ab}} \cong \mathbb{Z}^{2 g}$. By the previous lemma, the kernel $K$ of $\pi_{1}\left(X^{\text {aff }}\right) \rightarrow \pi_{1}(X)$ is isomorphic to $\mathbb{Z} / d \mathbb{Z}$. 
We plug this data into (8) and see that $H_{2}\left(\pi_{1}\left(X^{\text {aff }}\right), \mathbb{Z}\right)$ is an extension of $\pi_{1}(X)^{\mathrm{ab}} \otimes \mathbb{Z} / d \mathbb{Z}$ by $L:=\operatorname{ker}\left(H_{2}\left(\pi_{1}(X), \mathbb{Z}\right) \rightarrow \mathbb{Z} / d \mathbb{Z}\right)$. Since $H_{2}\left(\pi_{1}(X), \mathbb{Z}\right) \cong \mathbb{Z}$, the group $L$ is infinite cyclic, i.e., abstractly isomorphic to $\mathbb{Z}$. So we obtain a short exact sequence of Abelian groups

$$
0 \rightarrow(\mathbb{Z} / d \mathbb{Z})^{2 g} \rightarrow H_{2}\left(\pi_{1}\left(X^{\text {aff }}\right), \mathbb{Z}\right) \rightarrow \mathbb{Z} \rightarrow 0
$$

which splits since the quotient is free Abelian.

For a natural number $n \geq 1$ we define the homomorphism

$$
\begin{aligned}
& \kappa_{n}:(\mathbb{Z} / d \mathbb{Z})^{n} \quad \rightarrow \quad \mathbb{Z} / \operatorname{gcd}(d, k) \mathbb{Z}, \\
& \left(x_{1}, \ldots, x_{n}\right) \mapsto \quad \sum_{i=1}^{n} \bar{x}_{i}
\end{aligned}
$$

The following statement is now an easy application of Theorem 4.5] and Proposition 4.8 .

Proposition 5.10. For a generic projection from $X=C \times \mathbb{P}^{1}$ there exists a cyclic group $Z$ such that we obtain $\pi_{1}\left(X_{\text {gal }}\right) / C^{\text {proj }}$ as an extension

$$
0 \rightarrow\left((\mathbb{Z} / d \mathbb{Z})^{2 g} \oplus \mathbb{Z}\right) / Z \rightarrow \pi_{1}\left(X_{\text {gal }}\right) / C^{\text {proj }} \rightarrow G \rightarrow 1
$$

where $G$ is a central extension of the form

$$
0 \rightarrow \operatorname{ker} \kappa_{2 d k-1} \rightarrow G \rightarrow \mathcal{K}\left(\pi_{1}(C), 2 d k\right) \rightarrow 1
$$

Abelianising, we obtain an isomorphism

$$
H_{1}\left(X_{\text {gal }}, \mathbb{Z}\right) \overline{C^{\text {proj }}} \cong(\mathbb{Z} / \operatorname{gcd}(d, k) \mathbb{Z})^{2 d k-2} \oplus \mathbb{Z}^{2 g(2 d k-1)} .
$$

Remark 5.11. The results of [AG] and [AGTV] show that $C^{\text {aff }}$ and $C^{\text {proj }}$ are trivial in the case $g=1$ and $d=1$, i.e., Question 3.4 again has a positive answer. Also, in their computations it turns out that $Z$ kills the kernel of (10), i.e., $\pi_{1}\left(X_{\text {gal }}\right)$ is then isomorphic to $\mathcal{K}\left(\pi_{1}(X), 2 k\right) \cong \mathbb{Z}^{4 k-2}$.

\section{ACKNOWLEDGEMENTS}

This article extends the main results of the author's Ph.D. thesis [Li]. He thanks his supervisor Gerd Faltings for suggesting this interesting topic and the discussions on it. Also, the author thanks the Max-Planck-Institut in Bonn for hospitality and financial support. Finally, he thanks the referee for pointing out a few inaccuracies.

\section{REFERENCES}

[AGTV] M. Amram, D. Goldberg, M. Teicher, U. Vishne, The fundamental group of a Galois cover of $\mathbb{C P}^{1} \times \mathbb{T}$, Algebr. Geom. Topol. 2, 403-432 (2002). MR1917060 (2004a:14023)

[AG] M. Amram, D. Goldberg, Higher degree Galois covers of $\mathbb{C P}^{1} \times \mathbb{T}$, Algebr. Geom. Topol. 4, 841-859 (2004). MR2100683 (2005i:14016)

[Br] K. S. Brown, Cohomology of Groups, GTM 87, Springer (1982). MR672956 (83k:20002)

[Ch] D. Cheniot, Le théorème de Van Kampen sur le groupe fondamental du complementaire d'une courbe algébrique projective plane, Séminaire François Norguet 1970-73, LNM 409, 394-417 (1974). MR0369370 (51:5603)

[Fa] G. Faltings, A New Application of Diophantine Approximations, A Panorama in Number Theory or The View from Baker's Garden, ed. by G. Wüstholz, Cambridge University Press (2002), 231-246. MR1975455 (2004b:11100)

[FL] W. Fulton, R. Lazarsfeld, Connectivity and its applications in algebraic geometry, Springer LNM 862 (1981), 26-92. MR644817 (83i:14002)

[SGA1] A. Grothendieck, Revêtements étales et groupe fondamental, Séminaire de géométrie algébrique du Bois Marie 1960-61, Société Mathématique de France (2003). MR2017446 (2004g:14017) 
[Ku] V. S. Kulikov, On Chisini's Conjecture. II, arXiv:math/0610356 (2006), to appear in Izv. RAN. Ser. Mat.

[Li] C. Liedtke, On Fundamental Groups of Galois Closures of Generic Projections, Bonner Mathematische Schriften 367 (2004). MR2206239 (2007m:14023)

[Li2] C. Liedtke, Natural central extensions of groups, Groups Geom. Dyn. 2, 245-261 (2008). MR2393181

[Mi] Y. Miyaoka, Algebraic surfaces with positive indices, Proc. Symp. Katata/Jap. 1982, Prog. Math. 39, 281-301 (1983). MR728611 (85j:14067)

[Mo] B. Moishezon, Stable branch curves and braid monodromies, Springer LNM 862, 107-192 (1981). MR644819 (83c:14008)

[MoTe1] B. Moishezon, M. Teicher, Simply-connected algebraic surfaces of positive index, Invent. Math. 89, 601-643 (1987). MR903386 (88f:14037)

[MoTe2] B. Moishezon, M. Teicher, Galois coverings in the theory of algebraic surfaces, Proc. Symp. Pure Math. 46, 47-65 (1987). MR927973 (89h:14031)

[MoTe3] B. Moishezon, M. Teicher, Finite fundamental groups, free over $\mathbb{Z} / c \mathbb{Z}$, for Galois covers of $\mathbb{C P}^{2}$, Math. Ann. 293, No. 4, 749-766 (1992). MR1176029 (93i:14016)

[MTR] B. Moishezon, M. Teicher, A. Robb, On Galois covers of Hirzebruch surfaces, Math. Ann. 305, 493-539 (1996). MR1397434 (97h:14054)

[No] M. V. Nori, Zariski's conjecture and related problems, Ann. Sci. Éc. Norm. Sup. (4) 16, 305-344 (1983). MR732347 (86d:14027)

[Re] I. Reider, Vector bundles of rank 2 and linear systems on algebraic surfaces, Ann. Math. 127, 309-316 (1988). MR932299 (89e:14038)

[RTV] L. Rowen, M. Teicher, U. Vishne, Coxeter covers of the symmetric groups, J. Group Theory 8, No. 2, 139-169 (2005). MR2126726 (2006e:20073)

[Te] M. Teicher, New invariants for surfaces, Contemp. Math. 231, 271-281 (1999). MR:1707349 (2001c:14040)

Mathematisches Institut, Heinrich-Heine-Universität, 40225 Düsseldorf, Germany

E-mail address: liedtke@math.uni-duesseldorf.de

Current address: Department of Mathematics, Stanford University, 450 Serra Mall, Stanford, California 94305

E-mail address: liedtke@math.stanford.edu 